\title{
Uncertainty Propagation of a Multiscale Poromechanics-Hydration Model for Poroelastic Properties of Cement Paste at Early-Age
}

\author{
N. Venkovic ${ }^{\mathrm{a}}$, L. Sorelli ${ }^{\mathrm{a}, *}$, B. Sudret ${ }^{\mathrm{b}}$, T. Yalamas ${ }^{\mathrm{c}}$, R. Gagnéd \\ ${ }^{a}$ Département de Génie Civil, Université Laval, G1V 0A6 Québec, QC, Canada \\ ${ }^{b}$ ETH Zürich, Institute of Structural Engineering, \\ Chair of Risk, Safety E Uncertainty Quantification, CH-8093 Zürich, Switzerland \\ ${ }^{c}$ Phimeca Engineering, Centre d'Affaires du Zénith, 63800 Cournon, France \\ ${ }^{d}$ Département de Génie Civil, Université de Sherbrooke, J1K 2R1 Sherbrooke, QC, Canada
}

\begin{abstract}
The durability of concrete materials with regard to early-age volume changes and cracking phenomena depends on the evolution of the poroelastic properties of cement paste. The ability of engineers to control the uncertainty of the percolation threshold and the evolution of the elastic modulus, the Biot-Willis parameter and the skeleton Biot modulus is key for minimizing the vulnerability of concrete structures at early-age. This work presents original results on the uncertainty propagation and the sensitivity analysis of a multiscale poromechanics-hydration model applied to cement pastes of water-to-cement ratio of $0.40,0.50$ and 0.60 . Notably, the proposed approach provides poroelastic properties required to model the behavior of partially saturated aging cement pastes (e.g. autogenous shrinkage) and it predicts the percolation threshold and undrained elastic modulus in good agreement with experimental data. The development of a stochastic metamodel using polynomial chaos expansions allows to propagate the uncertainties of kinetic parameters of hydration, cement phase composition, elastic moduli and morphological parameters of the microstructure. The presented results show that the propagation does not magnify the uncertainty of the single poroelastic properties although, their correlation may amplify the variability of the estimates obtained from poroelastic state equations. In order to reduce the uncertainty of the percolation threshold and that of the poroelastic properties at early-age, engineers need to assess more accurately the apparent activation energy of calcium aluminate and, later on, of the elastic modulus of low density calcium-silicate-hydrate.
\end{abstract}

Keywords: Microporomechanics, Global sensitivity analysis, Sobol' variance decomposition, Elastic modulus, Biot-Willis parameter, Percolation threshold, Cement paste

\section{Introduction}

In poroelastic media such as concrete materials [1], the development of internal stresses due to drying and autogenous shrinkage and the risk of cracking significantly depend on the evolution of the poroelastic properties [2-4]. The ability of engineers to predict and control the variability of the percolation threshold, the elastic modulus, the Biot-Willis parameter and the skeleton Biot modulus is of prime importance to minimize the risk of cracking of concrete structures at early-age.

Recently, continuum micromechanics models have been used in many successful assessments of the effective mechanical properties of hardened cement-based materials [5-8]. The extension of these models to take into account the poroelastic behavior of concrete materials $[1,9,10]$ introduced several model applications [11-14]. At early-age, the microstructure evolves due to the hydration of anhydrous cement particles and additional chemical reactions. Bernard et al. [6] were the first to

\footnotetext{
${ }^{*}$ Corresponding author. Tel.: +1 418656 3060; fax: +1 4186563355

Email addresses: nicolas.venkovic.1@ulaval.ca (N. Venkovic), luca.sorelli.1@gci.ulaval.ca (L. Sorelli), sudret@ibk. baug.ethz.ch (B. Sudret), yalamas@phimeca.com (T. Yalamas), richard.gagne@usherbrooke.ca (R. Gagné)
}

model the evolution of the elastic modulus and Poisson's ratio of hydrating concrete materials by means of micromechanics. They proposed a hydration model and described the aging process by an evolution of the relative volumetric proportions of elementary phases with invariant mechanical properties. While providing good results for mid- to late-stages of hydration, this model lacked of precision at very early-age. Later on, Sanahuja et al. $[15,16]$ studied the impact of the shape of solid hydrates on the prediction of the solid percolation threshold and the elastic modulus of aging cement pastes. However, portlandite and ettringite were not considered among the hydration products responsible for setting in the model.

The input parameters of these deterministic models can be classified into four categories: the initial phase quantification, the kinetic parameters of hydration, the invariant elastic properties and the microstructural/morphological parameters. This information is uncertain and, as a consequence, the model responses can be considered as random variables. Berveiller et al. [17] first propagated uncertainty through a multistep micromechanics model of concrete. They predicted the variability of the elastic modulus and Poisson's ratio of cement paste by polynomial chaos expansions. The resulting variances were decomposed into Sobol' indices recovered by post-processing 
the polynomial expansions for much less calculation than what is required by Monte-Carlo simulation. Later on, Sudret et al. [18] used polynomial chaos expansions as stochastic metamodels in order to assess the full randomness of elastic properties at different scales of hardened concrete and perform sensitivity analyses from scale to scale. However, a sensitivity analysis of the poroelastic properties of cement paste over time has not yet been accomplished.

The purpose of this work is threefold: first, to provide a deterministic multiscale poromechanics-hydration model to predict the evolution of the Biot-Willis parameter, the skeleton Biot modulus, the Poisson's ratio, the elastic modulus and the percolation threshold; second, to improve the agreement between the prediction of the elastic modulus and experimental data by considering the effect of the shape of ettringite and portlandite particles on setting; third, to assess the uncertainty of these predictions at different stages of hydration and identify their greatest contributors among the input parameters. The originality of this work relies on the application of a non-intrusive method of uncertainty propagation to a microporomechanics-hydration model for cement pastes in order to assess the contribution of the material parameters to the variability of the macroscopic poroelastic properties over time. For the first time, this work presents the second central moment of the Biot-Willis parameter and the Biot skeleton modulus as functions of time.

The uncertainty propagation is performed by polynomial chaos expansion of the random model responses [19]. This approach has the advantage to give access to sensitivity information and to allow the computation of large samples of predictions for much less calculation than required by Monte-Carlo.

The paper is organized as follows: firstly, the materials studied in this work are presented; secondly, the deterministic multiscale poromechanics-hydration model is introduced; thirdly, the polynomial chaos expansion and the post-processing are explained; fourthly, the uncertainties of the input parameters are described; fifthly, the model is validated, the uncertainty is propagated, a sensitivity analysis is performed, the correlation between the poroelastic properties is investigated and the PDF of the elastic modulus is estimated at different timesteps.

\section{Material}

This work focuses on three cement pastes with different water-to-cement ratio $(w / c)$ of $0.40,0.50$ and 0.60 . The cement composition is an important factor that influences the chemistry of hydration and the properties of cement paste. The cement composition of this study is taken from the experimental study of Boumiz et al. [20, 21] on the elastic properties at early-age of cement-based materials. Two approaches can be used to describe the cement composition: the oxide composition and the chemical phase composition. The major oxides composition of this cement is presented in Table 1.

To model hydration, one needs to assess the phase composition. Cements are made of four major grinded clinker phases that react differently with water: tricalcium silicate, dicalcium silicate, tricalcium aluminate (aluminate) and tetracalcium aluminoferrite (ferrite). According to cement chem-
Table 1: Major oxides composition of cement PCCB9402 [20]

\begin{tabular}{lc}
\hline Oxyde & Mass fraction [\%] \\
\hline $\mathrm{CaO}$ & 66.23 \\
$\mathrm{SiO}_{2}$ & 21.68 \\
$\mathrm{Fe}_{2} \mathrm{O}_{3}$ & 0.29 \\
$\mathrm{Al}_{2} \mathrm{O}_{3}$ & 4.17 \\
$\mathrm{SO}_{3}$ & 3.54 \\
\hline
\end{tabular}

istry those are refered to as $\mathrm{C}_{3} \mathrm{~S}, \mathrm{C}_{2} \mathrm{~S}, \mathrm{C}_{3} \mathrm{~A}$ and $\mathrm{C}_{4} \mathrm{AF}$ where $\mathrm{C}=\mathrm{CaO}, \mathrm{A}=\mathrm{Al}_{2} \mathrm{O}_{3}, \mathrm{~F}=\mathrm{Fe}_{2} \mathrm{O}_{3}, \overline{\mathrm{S}}=\mathrm{SO}_{3}$ and $\mathrm{H}=\mathrm{H}_{2} \mathrm{O}$ so that $\mathrm{C}_{3} \mathrm{~S}=3 \mathrm{CaO} \cdot \mathrm{SiO}_{2}$ and so on. An amount of gypsum $\left(\mathrm{CS} \mathrm{H}_{2}\right)$ about $5 \%$ of the total mass of the system is usually added to these phases by cement producers.

Several methods can be used to determine the phase composition using the stoichiometry of those compounds and the respective amounts of the former oxides. The approach adopted in this study is a widely used method developped by Bogue [22]. It consists in a linear system of equations based on a quantitative oxide composition (see Table 1) under certain assumptions:

- the main phases of the cement are $\mathrm{C}_{3} \mathrm{~S}, \mathrm{C}_{2} \mathrm{~S}, \mathrm{C}_{3} \mathrm{~A}$ and $\mathrm{C}_{4} \mathrm{AF}$, to which are added some gypsum and free lime;

- all the $\mathrm{Fe}_{2} \mathrm{O}_{3}$ present in the system occurs as $\mathrm{C}_{4} \mathrm{AF}$;

- the remaining amount of $\mathrm{Al}_{2} \mathrm{O}_{3}$ occurs as $\mathrm{C}_{3} \mathrm{~A}$;

- the $\mathrm{CaO}$ occurs either as $\mathrm{C}_{3} \mathrm{~S}, \mathrm{C}_{2} \mathrm{~S}$, free lime or gypsum.

The mathematical formulation of the Bogue calculation is well documented and can be found in the book of Taylor [23]. We assume an amount of free lime equal to $1 \mathrm{~g} / 100 \mathrm{~g}$ of cement. The amount of calcium oxide that occurs as gypsum is fixed to $70 \%$ the mass of $\mathrm{SO}_{3}$.

\section{Multiscale poromechanics-hydration model}

The multiscale poromechanics-hydration model is applied in a two-step manner: a hydration model and a multiscale poromechanics model. The hydration model allows to define the evolution of the volumetric fractions of the invariant material phases contained in the aging cement paste. The multiscale poromechanics model is used to upscale the poroelastic properties based on a microstructure scheme.

\subsection{Hydration model}

The hydration model adopted in this work was proposed by Bernard et al. [6] and improved by Pichler et al. [11]. Following the pioneering work of Powers and Brownyard [24], it consists in stoichiometric and kinetic equations used to assess the evolution of volume fractions of hydration products and reactants through time. 


\subsubsection{Stoichiometry}

Independently from the rate of reaction, the amounts of reactants and products involved in cement hydration can be assessed by stoichiometric relations. Those reactions occur between the anhydrous compounds named above $\left(\mathrm{C}_{3} \mathrm{~S}, \mathrm{C}_{2} \mathrm{~S}, \mathrm{C}_{3} \mathrm{~A}, \mathrm{C}_{4} \mathrm{AF}\right.$, and $\mathrm{CS} \mathrm{H}_{2}$ ) and water to form hydration products. Some of the hydration products also react further on. Tennis and Jennings [25] proposed stoichiometric relations to describe this process:

$$
\begin{gathered}
2 \mathrm{C}_{3} \mathrm{~S}+10.6 \mathrm{H} \rightarrow \mathrm{C}_{3.4} \mathrm{~S}_{2} \mathrm{H}_{8}+2.6 \mathrm{CH} \\
2 \mathrm{C}_{2} \mathrm{~S}+8.6 \mathrm{H} \rightarrow \mathrm{C}_{3.4} \mathrm{~S}_{2} \mathrm{H}_{8}+0.6 \mathrm{CH} \\
\mathrm{C}_{3} \mathrm{~A}+3 \mathrm{C} \overline{\mathrm{S}} \mathrm{H}_{2}+26 \mathrm{H} \rightarrow \mathrm{C}_{6} \mathrm{AS}_{3} \mathrm{H}_{32} \\
2 \mathrm{C}_{3} \mathrm{~A}+\mathrm{C}_{6} \mathrm{~A}_{3} \mathrm{H}_{32}+4 \mathrm{H} \rightarrow 3 \mathrm{C}_{4} \mathrm{~A} \overline{\mathrm{S}} \mathrm{H}_{12} \\
\mathrm{C}_{4} \mathrm{AF}+2 \mathrm{CH}+10 \mathrm{H} \rightarrow 2 \mathrm{C}_{3}(\mathrm{~A}, \mathrm{~F}) \mathrm{H}_{6}
\end{gathered}
$$

Eq. (1) and Eq. (2) describe the formation of calcium-silicatehydrates $\left(\mathrm{C}_{3.4} \mathrm{~S}_{2} \mathrm{H}_{8}\right)$ and portlandite $(\mathrm{CH})$. Eq. (3) allows to quantify the amount of ettringite $\left(\mathrm{C}_{6} \overline{\mathrm{S}}_{3} \mathrm{H}_{32}\right)$, also noted $\mathrm{AFt}$, formed by hydration of gypsum and aluminate. Ettringite can react further with aluminate to form some monosulfoaluminate $\left(\mathrm{C}_{4} \mathrm{~A} \overline{\mathrm{S}} \mathrm{H}_{12}\right)$ also noted $\mathrm{AFm}$. The hydration of ferrite leads to the precipitation of hydrogarnet $\left(\mathrm{C}_{3}(\mathrm{~A}, \mathrm{~F}) \mathrm{H}_{6}\right)$.

\subsubsection{Kinetic}

The mass exchanges described by stoichiometry occur at different rates depending on the clinker phase involved in the reaction. The advancement of the hydration process of a sole clinker phase is expressed by means of a hydration degree:

$$
\xi_{X}(t) \equiv 1-\frac{m_{X}(t)}{m_{X, 0}}
$$

Where the hydration degree $\xi_{X}$ refers to the relative amount of reactant consumed $m_{X}$ compared to the initial amount $m_{X, 0}$ present in the system. The overall hydration degree $\xi$ is obtained by summing the hydration degrees of all the anhydrous phases weighted by their respective initial weight fraction. The kinetics of hydration of the four major clinker phases is described by relationships that link the reaction rate $\mathrm{d} \xi_{X} / \mathrm{d} t$ to the affinity $A\left(\xi_{X}\right)$ referred to as the driving force of the hydration reaction. In a first order approach, we disregard the chemomechanical couplings which are of secondary importance for normal conditions of temperature and pressure. The normalized affinity is expressed as follows:

$$
\tilde{A}\left(\xi_{X}\right)=\tau_{X, 0} \exp \left[\frac{E_{a, X}}{\mathscr{R}}\left(\frac{1}{T_{0}}-\frac{1}{T}\right)\right] \frac{\mathrm{d} \xi_{X}}{\mathrm{~d} t}
$$

Where $E_{a, X}$ is the apparent activation energy for the hydration of a phase $X$ among the major clinker compounds and $\mathscr{R}$ is the universal gas constant. The characteristic time $\tau_{X, 0}$ is defined at a reference temperature $T_{0}$ and depends on the hydration process. Three main reaction processes can be considered to describe the hydration of a cement mixture: dissolution, growth and nucleation and diffusion. Dissolution is the first reaction to occur. It can be described with a normal affinity $\tilde{A}\left(\xi_{X}\right)$ of 1 and a characteristic time $\tau_{X, 0}$ equal to $t_{X, 0} / \xi_{X, 0}$. The end of dissolution is marked by a threshold degree of hydration $\xi_{X, 0}$ for each of the main clinker phases.

Growth and nucleation follow the dissolution. This process is well described by the phase evolution model of Avrami [26]. The corresponding normalized affinity is:

$$
\tilde{A}^{A}\left(\xi_{X}\right)=\frac{1-\left\langle\xi_{X}-\xi_{X, 0}\right\rangle}{\left[-\ln \left(1-\left\langle\xi_{X}-\xi_{X, 0}\right\rangle\right)\right]^{(1 / \kappa)-1}}
$$

Where the operator $\langle\cdot\rangle$ is such that $\langle x\rangle=1 / 2(x+|x|) \forall x \in \mathbb{R}$. The characteristic time $\tau_{x, 0}$ related to this kinetic law is equal to $1 /\left(\kappa_{X} k_{X}\right)$ where $\kappa_{X}$ is the reaction order and $k_{X}$, a constant rate.

Diffusion occurs as a late hydration process beyond a threshold degree $\xi_{x}^{*}$ defined for every main clinker phase. The normalized affinity associated with this process is:

$$
\tilde{A}^{D}\left(\xi_{X}\right)=\frac{\left(1-\xi_{X}\right)^{\frac{2}{3}}}{\left(1-\xi_{X}^{*}\right)^{\frac{1}{3}}-\left(1-\xi_{X}\right)^{\frac{1}{3}}}
$$

Thanks to the work of Fujii and Kondo [27], the characteristic time $\tau_{X, 0}$ of this kinetic mechanism can be expressed by $R^{2} /\left(3 D_{x}\right)$ where $R$ is the initial mean radius of the anhydrous cement particles and $D_{X}$, the coefficient of diffusion of dissolved ions through existing layers of hydration products towards the remaining anhydrous phase $X$.

Because the hydration of aluminate follows two stoichiometric reactions, an order of priority needs to be specified between Eq. (3) and Eq. (4). Aluminate reacts primarily with gypsum to form ettringite as described by Eq. (3). Once all the gypsum is consumed, it can react with ettringite to form monosulfoaluminate with respect to Eq. (4). Therefore, a very simplistic interaction between chemical reactions is assumed. Although some models exist that explicitly support the effect of complex phenomena such as water accessibility to anhydrous phases [28] and confined growth of hydration product [29,30], it is beyond the scope of this study to provide such a refined description of the kinetics.

It is assumed that the diffusion process leads to the precipitation of calcium-silicate-hydrates $(\mathrm{C}-\mathrm{S}-\mathrm{H})$ that are denser than those associated with dissolution and nucleation [6]. Hence, a distinction is made between the C-S-H precipitated either during the first hydration processes or by diffusion. With respect to the nomenclature introduced by Jennings [31], the first ones are referred to as low density (LD) C-S-H and the last ones as high density (HD) C-S-H.

\subsubsection{Volume fractions}

The volumes $V_{X}$ of the main clinker phases can be computed as functions of time equal to $m_{X, 0} \xi_{X}(t) / \rho_{X}$ where the molar masses $\mathcal{M}_{X}$ and densities $\rho_{X}$ are given by Tennis and Jennings [25]. The hydration degrees $\xi_{X}(t)$ are obtained from the solution to Eq. (7) with respect to the appropriate expression of the normalized affinity of each kinetic process. It is assumed that the sample is hydrated with an infinite supply of water so that the volumes of hydration products can be computed as functions of time governed by the remaining amounts of major clinker phases: 


$$
V_{P}(t)=\frac{\mathcal{M}_{P}}{\mathcal{M}_{X}} \frac{\rho_{X}}{\rho_{P}} m_{X, 0} n_{P / X} \xi_{X}(t)
$$

where the index $P$ refers to the hydration product and $n_{P / X}$ is the number of moles of product generated by hydration of one mole of anhydrous cement phase $X$. The stoichiometric ratios $n_{P / X}$ are straightforwardly obtained from Eqs. (1)-(5). The volume fractions of reactants $f_{X}(t)$ and hydration products $f_{P}(t)$ are obtained by normalization of the respective volumes $V_{X}$ and $V_{P}$ with the total volume at time $t$.

\subsection{Multiscale poromechanics model}

The multiscale poromechanics model provides the BiotWillis parameter, the skeleton Biot modulus, the Poisson's ratio, the elastic modulus and the percolation threshold of cement paste as functions of volume fractions which are obtained from the hydration model.

The approach adopted is inspired from underlying works in microporomechanics $[1,9,10]$ and model applications to earlyage cement-based materials $[6,15,16]$. However, the proposed model assumes different hypotheses with respect to the work of Sanahuja et al. [15], such as: (i) a spherical elementary C-S-H particle at the nanometer scale. As demonstrated by Sanahuja et al [32], the shape of particles of polycrystals with packing density greater than $60 \%$ (as LD and HD C-S-H) is a second order parameter; (ii) a larger number of anhydrous phases and hydration products; (iii) spherical particles of HD C-S-H embedded into a matrix of LD C-S-H. Although the observed morphology of C-S-H at the scale of several hundreds of nanometers is often refered to as fibrillar, it varies over time [33] and depends on drying conditions that provoke the rearrangement of the elementary C-S-H particles [34]. As it is difficult to define a precise morphology that will correspond to the shape of LD and HD C-S-H in any conditions, the same representation that Bernard et al. [6] is adopted (see Fig. 1); (iv) non-spherical inclusions of portlandite and ettringite. Crystals of portlandite and ettringite exhibit strongly aspherical shapes and contribute to the setting of cement paste [23]; (v) a fixed porosity of LD C-S-H.

The model is developed as follows: firstly, the microstructure of cement paste is represented in terms of elementary phases of invariant mechanical properties. A representative elementary volume (REV) [35] that contains qualitative and morphological information is drawn for every characteristic length scale of the material. These elements are significantly smaller than the size of the structure and larger than the inhomogeneities they contain; secondly, the microscopic mechanical responses of the cement paste subjected to prescribed macroscopic boundary conditions are expressed by means of localization tensors; thirdly, the general macroscopic response of the system is expressed in terms of state equations obtained by homogenization of the local reactions. The investigated poroelastic properties are recovered from these resulting equations.

\subsubsection{Microstructure representation}

Converging efforts of experimental characterization of mechanical properties at nanoscale $[5,7]$ and modeling of calcium- silicate-hydrates $[31,36]$ have led to a multiscale representation of heterogeneity in cement-based materials. A microstructure of cement paste can be described over three length scales [1]. As presented in Fig. 1, those are, from the coarsest to the finest: the cement paste itself (level II), the aging C-S-H (level I) and the scale of preferential invariant densities of hydrates (level0). The qualitative phase composition of each scale and the corresponding essential morphological information are communicated below.
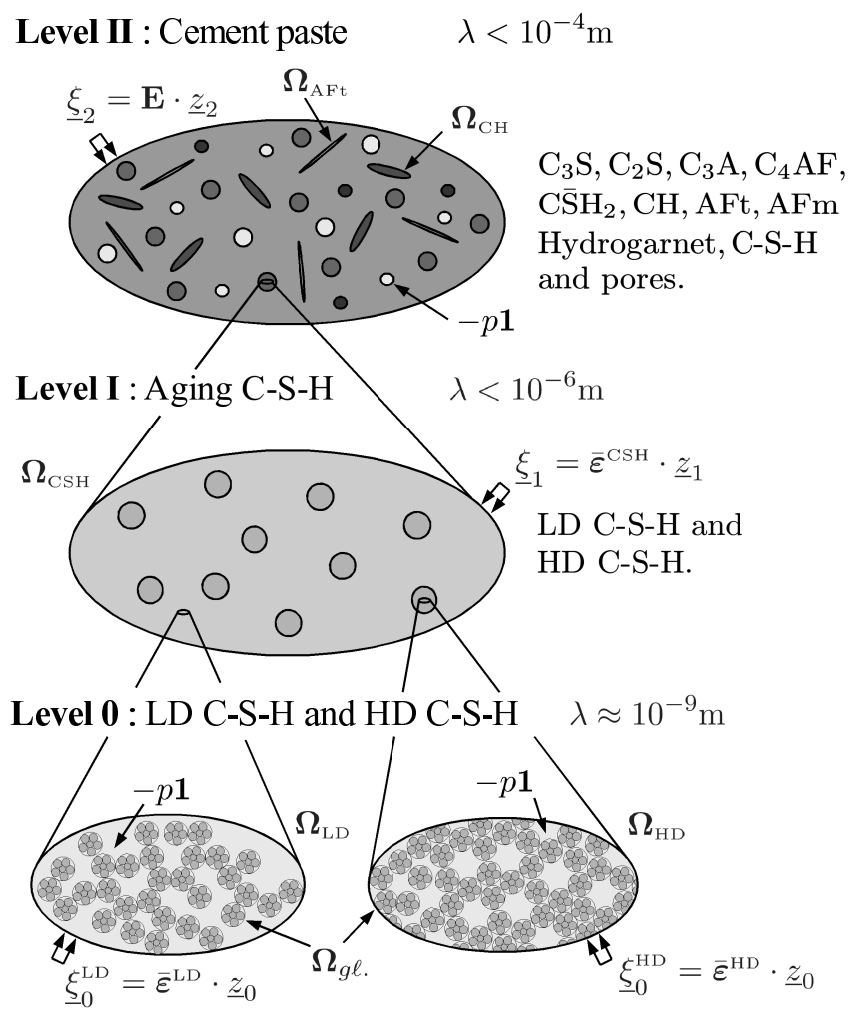

Figure 1:

Level II is representative of heterogeneities of characteristic lengths smaller than $10^{-4} \mathrm{~m}$. The phases at this scale are the anhydrous cement particles, hydration products and pores. The anhydrous phases $\left(\mathrm{C}_{3} \mathrm{~S}, \mathrm{C}_{2} \mathrm{~S}, \mathrm{C}_{3} \mathrm{~A}, \mathrm{C}_{4} \mathrm{AF}\right.$, and $\left.\mathrm{CS} \mathrm{S}_{2}\right)$ are not very sharp [23] and are usually considered as spherical inclusions $[6,15]$. The main non-porous elementary hydration products are portlandite, AFt, AFm and hydrogarnet. The shape of AFm and hydrogarnet is not well defined and could even change during hydration [23]. Due to lack of information and their small volume fractions, they are here simply represented by spherical inclusions $[11,37]$. Portlandite can be present in great amounts in form of platy crystals which are here represented by oblate ellipsoids [37]. AFt crystals exhibit very sharp rod-like shapes and can be modeled by prolate ellipsoids [37]. The pores are part of the capillary porosity and remain filled of water, they are considered as spherical inclusions. Calcium-silicatehydrates are present as a sole homogeneous aging phase. At this scale, C-S-H particles can either be considered as needles $[38,39]$ or as spherical inclusions [6, 7]. This is a long-standing and still open scientific debate. Here, the C-S-H particles are 
considered spherical.

When hydration starts, the mixture contains a greater amount of liquid than solids and the paste behaves like a viscous fluid. As long as enough hydration product is precipitated to ensure a contact between solid particles, the paste sets and exhibits an increasing stiffness as a function of time. As stated by Bernard et al. [6], this is the scale of solid percolation [40].

Level I is representative of heterogeneities of characteristic length smaller than $10^{-6} \mathrm{~m}$. This is the smallest scale that can be characterized by current nanoindentation techniques due to roughness limitations [41]. According to the colloidal model of calcium-silicate-hydrates proposed by Jennings [31, 36], these phases are the hydration products that constitute the actual aging C-S-H: low density and high density C-S-H, also refered to as LD and HD C-S-H or outer and inner products [42]. The invariance of the properties of these phases was the major finding of Constantinides [10] confirmed by Vandamme [43]. Earlyhydration is characterized by nucleation and growth of LD CS-H. HD C-S-H precipitates later, within smaller regions confined by low density product. Hence, the high density calciumsilicate-hydrates are represented by spherical inclusions embedded into a matrix of low density hydration product.

Level 0 is representative of heterogeneities of characteristic length about $10^{-9} \mathrm{~m}$. Two REV's are drawn in order to describe both types of calcium-silicate-hydrates. According to the colloidal model of Jennings [31, 36], LD and HD C-S-H are constituted of the same spherical C-S-H solid particles. These particles, referred to as globules (with the superscript ${ }^{\ell \ell}$ ), are packed at different densities with respect to each of the C-S-H phases. The corresponding gel porosities $\phi_{\mathrm{LD}}$ and $\phi_{\mathrm{HD}}$ were estimated to $37.3 \%$ and $23.7 \%$ and remain filled by water $[1,31]$.

Three position vectors $z_{k}$ with $k=0,1,2$ are defined over the REV's drawn at levels $0, \overline{\mathrm{I}}$ and II. An uniform strain boundary condition is applied on the REV at level II so that the displacement $\underline{\xi}_{2}$ is equal to $\mathbf{E} \cdot \underline{z}_{2}$ where $\mathbf{E}$ is the macroscopic uniform strain tensor. The REV's defined at smaller scales are subjected to the same kind of boundary conditions induced by local mechanical responses developed at relative upper scales. A pressure $p$ is applied in both interconnecting porosities defined at scale 0 and II.

\subsubsection{Localization}

At level I, the poroelastic behavior of the LD and HD C-S$\mathrm{H}$ phases is described by means of classical state equations of microporomechanics $[1,10,44]$. The mean stresses $\bar{\sigma}^{Y}$ induced by the prescribed strains $\overline{\boldsymbol{\varepsilon}}^{Y}$ and the pore pressure $p$ are related as follows:

$$
\overline{\boldsymbol{\sigma}}^{Y}=\mathbb{C}_{Y}: \overline{\boldsymbol{\varepsilon}}^{Y}-\mathbf{B}_{Y} p
$$

where $Y$ stands either for LD or HD C-S-H. The overbar operator is defined so that $\bar{a}^{i}$ refers to the volume average of $a$ over the phase $i$. The stiffness tensors $\mathbb{C}_{Y}$ are known. The contribution of the pore pressure on the stress states of these REV's is quantified by the Biot tensors $\mathbf{B}_{\mathrm{Y}}$ which can be readily calculated by stress average as:

$$
\mathbf{B}_{Y}=\mathbf{1}:\left[\mathbb{I}-\left(1-\phi_{Y}\right){\overline{\mathbb{A}\left(\underline{z}_{0}\right)}}^{g \ell}\right]
$$

where $\phi_{Y}$ refers to the porosities of LD and HD C-S-H, $\mathbf{1}$ is the second order identity tensor, and $\mathbb{I}$ is the fourth order identity tensor. The localization tensor ${\overline{\mathbb{A}\left(z_{0}\right)}}^{g \ell}$ would concentrate the totality of the strain $\overline{\boldsymbol{\varepsilon}}^{Y}$ on the globules of the corresponding phase $Y$ if the pore pressure was equal to zero.

The second poromechanical state equation expresses the Lagrangian porosity change as follows:

$$
\left(\phi-\phi_{0}\right)_{Y}=\mathbf{B}_{Y}: \overline{\boldsymbol{\varepsilon}}^{Y}+\frac{1}{N_{Y}} p
$$

where $N_{Y}$ 's are skeleton Biot moduli:

$$
\frac{1}{N_{Y}}=\mathbf{1}:\left(1-\phi_{Y}\right) \mathbb{S}_{g \ell}:\left(\mathbf{1}-\mathbf{1}:{\overline{\mathbb{A}\left(\underline{z}_{0}\right)}}^{g \ell}\right)
$$

The index $Y$ stands for LD and HD C-S-H and the compliance tensor of the globules $\mathbb{S}_{g \ell}$ is known.

The strains $\overline{\boldsymbol{\varepsilon}}^{Y}$ developed at scale 0 are due to the combined effect of the pore pressure $p$ and the mean strain $\overline{\boldsymbol{\varepsilon}}^{\mathrm{csH}}$ applied over the aging C-S-H at scale I. Thanks to linear elasticity, this problem can be decomposed into super-imposable loading cases: first, the strain $\bar{\varepsilon}^{\mathrm{csH}}$ is applied and the mean stresses ${\overline{\sigma^{\prime}}}^{{ }^{\prime}}$ and mean strains ${\overline{\boldsymbol{\varepsilon}^{\prime}}}^{Y}$ occur at level 0 . Second, the pore pressure $p$ is applied and the mean stresses ${\overline{\sigma^{\prime \prime}}}^{Y}$ and mean strains ${\overline{\varepsilon^{\prime \prime}}}^{Y}$ are developed. The application of Levin's theorem [44-46] provides the following relation:

$$
\sum_{Y=L D, H D} f_{Y}{\overline{\sigma^{\prime \prime}}}^{Y}:{\overline{\boldsymbol{\varepsilon}^{\prime}}}^{Y}=\left[\sum_{Y=L D, H D} f_{Y}{\overline{\bar{\sigma}^{\prime \prime}}}^{Y}\right]: \overline{\boldsymbol{\varepsilon}}^{\mathrm{CSH}}
$$

where the strains ${\overline{\boldsymbol{\varepsilon}^{\prime}}}^{Y}$ developed under the first loading case are of the form ${\overline{\mathbb{A}\left(z_{1}\right)}}^{Y}: \overline{\boldsymbol{\varepsilon}}^{\mathrm{CSH}}$. The relations between stress and strain developed under the second loading case are determined with respect to Eq. (11) and the above equation is recast as:

$$
p \sum_{Y=L D, H D} f_{Y} \mathbf{B}_{Y}:\left({\overline{\mathbb{A}\left(\underline{z}_{1}\right)}}^{Y}-\mathbb{I}\right)=\sum_{Y=L D, H D} f_{Y} \mathbb{C}_{Y}:{\overline{\bar{\varepsilon}^{\prime \prime}}}^{Y}:\left({\overline{\mathbb{A}\left(\underline{z}_{1}\right.}}^{Y}-\mathbb{I}\right)
$$

A second use of Levin's theorem leads to the following equation:

$$
\sum_{Y=L D, H D} f_{Y} \mathbb{C}_{Y}:{\overline{\mathbb{A}\left(z_{1}\right)}}^{Y}: \bar{\varepsilon}^{\mathrm{CSH}}:{\overline{\varepsilon^{\prime \prime}}}^{Y}=0
$$

An expression for ${\overline{\varepsilon^{\prime \prime}}}^{Y}$ is obtained from Eqs. (16) and (17) and the total strains $\overline{\boldsymbol{\varepsilon}}^{Y}$ are recovered by summing the strains developed under the first and second loading cases:

$$
\overline{\boldsymbol{\varepsilon}}^{Y}={\overline{\mathbb{A}\left({\underline{z_{1}}}^{\prime}\right.}}^{Y}: \overline{\boldsymbol{\varepsilon}}^{\mathrm{CSH}}+\mathbb{S}_{Y}: \mathbf{B}_{Y}:\left({\overline{\mathbb{A}\left({\underline{z_{1}}}^{Y}\right.}}^{Y}-\mathbb{I}\right) p
$$

where the compliance tensors $\mathbb{S}_{Y}$ are the inverses of the stiffness tensors $\mathbb{C}_{Y}$.

At level II, the strain acting over the aging C-S-H is due to the pore pressure $p$ and the macroscopic strain $\mathbf{E}$ applied over the cement paste. Similarly, the situation is decomposed into 
super-imposable loading cases: first, the strain $\mathbf{E}$ is applied and a mean stress $\overline{\sigma^{\prime}}{ }^{\mathrm{CSH}}$ and mean strain $\overline{\boldsymbol{\varepsilon}}^{\mathrm{CSH}}$ occur at level I; second, the pore pressure $p$ is applied and the mean stress ${\overline{\sigma^{\prime \prime}}}^{\mathrm{CSH}}$ and mean strain ${\overline{\varepsilon^{\prime \prime}}}^{\mathrm{CSH}}$ are developed. A first application of Levin's theorem gives the following relation:

$$
\begin{aligned}
-\varphi_{2} p \mathbf{1}+ & \left.f_{\mathrm{CSH}}{\overline{\boldsymbol{\sigma}^{\prime \prime}}}^{\mathrm{CSH}}+\sum_{s \in \mathscr{A}_{s}} f_{s}{\overline{\boldsymbol{\sigma}^{\prime \prime}}}^{s}\right]: \mathbf{E}=-\varphi_{2} p \mathbf{1}:{\overline{\mathbb{A}\left(\underline{z_{2}}\right)}}^{p}: \mathbf{E} \\
& +f_{\mathrm{CSH}}\left[\mathbb{C}_{\mathrm{CSH}}:{\overline{\boldsymbol{\varepsilon}^{\prime \prime}}}^{\mathrm{CSH}}-\mathbf{B}_{\mathrm{CSH}} p\right]:{\overline{\mathbb{A}\left(\underline{z}_{2}\right)}}^{\mathrm{CSH}}: \mathbf{E} \\
& +\sum_{s \in \mathscr{A}_{s}} f_{s} \mathbb{C}_{s}:{\overline{\boldsymbol{\varepsilon}^{\prime \prime}}}^{s}:{\overline{\mathbb{A}\left(\underline{z}_{2}\right)}}^{s}: \mathbf{E}
\end{aligned}
$$

where $\mathscr{A}_{S}$ is the set of non-porous solid phases of the REV at level II, $\varphi_{2}$ is the porosity at the same scale and the Biot tensor $\mathbf{B}_{\mathrm{CSH}}$ is defined to simplify the formulation [10]:

$$
\mathbf{B}_{\mathrm{CSH}}=f_{\mathrm{LD}}^{*}{\overline{\mathbb{A}\left(\underline{z}_{1}\right)}}^{\mathrm{LD}}: \mathbf{B}_{\mathrm{LD}}+f_{\mathrm{HD}}^{*}{\overline{\mathbb{A}\left(\underline{z}_{1}\right)}}^{\mathrm{HD}}: \mathbf{B}_{\mathrm{HD}}
$$

where $f_{\mathrm{LD}}^{*}=f_{\mathrm{LD}} /\left(f_{\mathrm{LD}}+f_{\mathrm{HD}}\right)$ and $f_{\mathrm{HD}}^{*}=f_{\mathrm{HD}} /\left(f_{\mathrm{LD}}+f_{\mathrm{HD}}\right)$ so that $f_{\mathrm{LD}}^{*}+f_{\mathrm{HD}}^{*}=1$.

The stiffness tensor $\mathbb{C}_{\mathrm{CSH}}$ of the aging $\mathrm{C}-\mathrm{S}-\mathrm{H}$ is:

$$
\mathbb{C}_{\mathrm{CSH}}=f_{\mathrm{LD}}^{*} \mathbb{C}_{\mathrm{LD}}:{\overline{\mathbb{A}\left(\underline{z}_{1}\right)}}^{\mathrm{LD}}+f_{\mathrm{HD}}^{*} \mathbb{C}_{\mathrm{HD}}:{\overline{\mathbb{A}\left(\underline{z}_{1}\right)}}^{\mathrm{HD}}
$$

Because the volume average of the localization tensors over a $\mathrm{REV}$ is equal to the fourth order unity tensor, the concentration tensor of the porosity of level II can be recast in:

$$
{\overline{\mathbb{A}\left(\underline{z}_{2}\right)}}^{p}=\frac{1}{\varphi_{2}}\left[\mathbb{I}-f_{\mathrm{CSH}}{\overline{\mathbb{A}\left(\underline{z}_{2}\right)}}^{\mathrm{CSH}}-\sum_{s \in \mathscr{A}_{s}} f_{s}{\overline{\mathbb{A}\left(\underline{z}_{2}\right)}}^{s}\right]
$$

A second application of Levin's theorem leads to the following equation:

$$
\sum_{s \in \mathscr{A}_{s}} f_{s} \mathbb{C}_{s}:{\overline{\mathbb{A}\left(\underline{z}_{2}\right)}}^{s}: \mathbf{E}:{\overline{\boldsymbol{\varepsilon}^{\prime \prime}}}^{s}+f_{\mathrm{CSH}} \mathbb{C}_{\mathrm{CSH}}:{\overline{\mathbb{A}\left(\underline{z}_{2}\right)}}^{\mathrm{CSH}}: \mathbf{E}:{\overline{\boldsymbol{\varepsilon}^{\prime \prime}}}^{\mathrm{CSH}}=0
$$

The total strain developed over the aging C-S-H can then be recovered from Eqs. (19), (22) and (23).

$$
\overline{\boldsymbol{\varepsilon}}^{\mathrm{CSH}}={\overline{\mathbb{A}\left(\underline{z}_{2}\right)}}^{\mathrm{CSH}}: \mathbf{E}+\mathbb{S}_{\mathrm{CSH}}:\left(\mathbf{1}-\mathbf{B}_{\mathrm{CSH}}\right):\left({\overline{\mathbb{A}\left(\underline{z}_{2}\right)}}^{\mathrm{CSH}}-\mathbb{I}\right) p
$$

The mean strain applied over every non-porous phase of scale II reduces to:

$$
\overline{\boldsymbol{\varepsilon}}^{s}={\overline{\mathbb{A}\left(\underline{z}_{2}\right)}}^{s}: \mathbf{E}+\mathbb{S}_{s}: \mathbf{1}:\left({\overline{\mathbb{A}\left(\underline{z}_{2}\right)}}^{s}-\mathbb{I}\right) p
$$

The mean strain applied over the capillary porosity is then equal to:

$$
\overline{\boldsymbol{\varepsilon}}^{p}=-\frac{1}{\varphi_{2}}\left[f_{\mathrm{CSH}} \overline{\boldsymbol{\varepsilon}}^{\mathrm{CSH}}+\sum_{s \in \mathscr{A}_{s}} f_{s} \overline{\boldsymbol{\varepsilon}}^{s}\right]
$$

The localizer tensor for an ellipsoidal inclusion in a medium subjected to uniform boundary conditions assessed from the solution of Eshelby [47]. The assumption is made that, for each phase $i$ of the level $\mathrm{k}$, the inclusions are randomly oriented in the REV with respect to a uniform distribution. Then, the strain concentration tensor ${\overline{\mathbb{A}\left(z_{k}\right)}}^{i}$ is obtained from the following space average over Euler angles [16]:

$$
{\overline{\mathbb{A}\left(\underline{z}_{k}\right)}}^{i}=\int_{\phi=0}^{2 \pi} \int_{\theta=0}^{\pi}\left[\mathbb{I}+\mathbb{P}_{i}(\theta, \phi):\left(\mathbb{C}_{0}-\mathbb{C}_{i}\right)\right]^{-1} \frac{\sin \theta}{4 \pi} \mathrm{d} \theta \mathrm{d} \phi
$$

The Hill polarization tensor $\mathbb{P}_{i}(\theta, \phi)$ depends on the shape of the inclusions of $\boldsymbol{\Omega}_{i}$, their orientation and the stiffness tensor $\mathbb{C}_{0}$ of the reference medium. Every polarization tensor is related to an Eshelby tensor $\mathbb{S}_{i}^{e s h}$ by the relation $\mathbb{S}_{i}^{e s h}=\mathbb{P}_{i}: \mathbb{C}_{0}$. The form of the Eshelby tensor is widely documented in textbooks on micromechanics [48]. The only missing parameter is the aspect ratio defined as the ratio of the length of the symmetry axis and the diameter in the symmetry plane. For oblates, spheres and prolates it is respectively smaller than 1 , equal to 1 and greater than 1. The double integration of Eq. (27) is performed with respect to the approximation of Stroud [39, 49].

A reference phase $\boldsymbol{\Omega}_{0}$ is defined in function of the morphology and the homogenization scheme adopted for each REV. Two homogenization schemes are considered: the self consistent scheme and the Mori-Tanaka scheme [50]. The MoriTanaka scheme is typical for media with a strong matrixinclusion morphology and considers the embedding phase as the reference medium. The self consistent scheme is appropriate for the representation of polydisperse granular media. It considers the resulting homogenized medium as a reference so that it involves an implicit formulation of the auxiliary problem. The self consistent scheme is well-suited to capture the solid percolation threshold of the REV drawn at level II [6]. However, as it was pointed out by different authors for low water-to-cement ratios, additional precautions must be taken into account to ensure the accuracy of the prediction of the solid percolation threshold $[6,14,15,51]$. In particular, Sanahuja et al. [15] considered LD C-S-H particles as platelets (oblates) with an aspect ratio varying in function of the water-to-cement ratio. On the other hand, Pichler et al. [39, 51] represented $\mathrm{C}-\mathrm{S}-\mathrm{H}$ as needle-like particles independently of the water-tocement ratio. Although both of these approaches were successfully applied, they were adapted to specific representations of the microstructure that differ from the ones adopted by Bernard et al. [6] and presented on Fig. 1. The self consistent scheme is also considered to model the nanogranular colloidal LD and HD C-S-H at scale 0 [52]. The Mori-Tanaka scheme is adopted to take into account the strong matrix-inclusion morphology of the aging C-S-H. The LD C-S-H is then referred to as the reference medium of scale I.

\subsubsection{Homogenization}

The macroscopic resulting stress tensor $\Sigma$ can be obtained by volumetric average of the localized resulting stresses:

$$
\boldsymbol{\Sigma}=\sum_{s \in \mathscr{A}_{s}} f_{s} \mathbb{C}_{s}: \overline{\boldsymbol{\varepsilon}}^{s}+f_{\mathrm{CSH}} \mathbb{C}_{\mathrm{CSH}}: \overline{\boldsymbol{\varepsilon}}^{\mathrm{CSH}}-\left(f_{\mathrm{CSH}} \mathbf{B}_{\mathrm{CSH}}+\varphi_{2} \mathbf{1}\right) p
$$


The state equation (28) can be recast in:

$$
\boldsymbol{\Sigma}=\mathbb{C}: \mathbf{E}-\mathbf{B} p
$$

where $\mathbb{C}$ is the homogenized stiffness tensor:

$$
\mathbb{C}=\sum_{s \in \mathscr{A}_{s}} f_{s} \mathbb{C}_{s}:{\overline{\mathbb{A}\left(\underline{z}_{2}\right)}}^{s}+f_{\mathrm{CSH}} \mathbb{C}_{\mathrm{CSH}}:{\overline{\mathbb{A}\left(\underline{z}_{2}\right)}}^{\mathrm{CSH}}
$$

The effective Biot tensor of the cement paste, $\mathbf{B}$, is expressed by:

$$
\begin{aligned}
\mathbf{B}= & \mathbf{1}:\left[\mathbb{I}-\sum_{s \in \mathscr{A}_{s}} f_{s}{\overline{\mathbb{A}\left(\underline{z}_{2}\right)}}^{s}-f_{\mathrm{CSH}}{\overline{\mathbb{A}\left(\underline{z}_{2}\right)}}^{\mathrm{CSH}}\right] \\
& +f_{\mathrm{CSH}}{\overline{\mathbb{A}\left(\underline{z}_{2}\right)}}^{\mathrm{CSH}}: \mathbf{B}_{\mathrm{CSH}}
\end{aligned}
$$

The total Lagrangian change in porosity is formulated as follows:

$$
\phi-\phi_{0}=\varphi_{2} \operatorname{tr} \bar{\varepsilon}^{p}+f_{\mathrm{CSH}}\left[f_{\mathrm{LD}}^{*}\left(\phi-\phi_{0}\right)_{\mathrm{LD}}+f_{\mathrm{HD}}^{*}\left(\phi-\phi_{0}\right)_{\mathrm{HD}}\right]
$$

where the first term represents the change in the capillary porosity (level II) and the second one represents the total change in the gel porosity (level 0 ). This state equation can be recast in:

$$
\phi-\phi_{0}=\mathbf{B}: \mathbf{E}+\frac{1}{N} p
$$

where $N$ is the skeleton Biot modulus. It exhibits two components, for the two scales where the pore pressure $p$ is applied:

$$
\frac{1}{N}=\frac{1}{N_{0}}+\frac{1}{N_{2}}
$$

The contribution of the pressure applied in the gel porosity on the total change in porosity can be quantified with $N_{0}$ :

$$
\begin{aligned}
\frac{1}{N_{0}}= & f_{\mathrm{CSH}}\left(\mathbf{1}-\mathbf{B}_{\mathrm{CSH}}\right):\left[\mathbb{S}_{\mathrm{CSH}}: \mathbf{B}_{\mathrm{CSH}}:\left({\overline{\mathbb{A}\left(\underline{z}_{2}\right)}}^{\mathrm{CSH}}-\mathbb{I}\right)\right] \\
& +f_{\mathrm{CSH}}\left(\frac{f_{\mathrm{LD}}^{*}}{N_{\mathrm{LD}}}+\frac{f_{\mathrm{HD}}^{*}}{N_{\mathrm{HD}}}\right)
\end{aligned}
$$

Likewise, the contribution of the pressure applied in the capillary pores on the total change in porosity can be quantified with $N_{2}$ :

$$
\begin{aligned}
\frac{1}{N_{2}}= & f_{\mathrm{CSH}}\left(\mathbf{B}_{\mathrm{CSH}}-\mathbf{1}\right):\left[\mathbb{S}_{\mathrm{CSH}}: \mathbf{1}:\left({\overline{\mathbb{A}\left(\underline{z}_{2}\right)}}^{\mathrm{CSH}}-\mathbb{I}\right)\right] \\
& +\mathbf{1}:\left\{\sum_{s \in \mathscr{A}_{s}} f_{S}\left(\mathbf{B}_{\mathrm{CSH}}-\mathbf{1}\right):\left[\mathbb{S}_{s}: \mathbf{1}:\left(\mathbb{I}-{\overline{\mathbb{A}\left(\underline{z}_{2}\right)}}^{s}\right)\right]\right\}
\end{aligned}
$$

In the absence of fluid mass exchange, the undrained stiffness tensor can be evaluated by [44]:

$$
\mathbb{C}^{u}=\mathbb{C}+M \mathbf{B} \otimes \mathbf{B}
$$

where the Biot modulus, $M$, is a function of the skeleton Biot modulus $N$ and the total porosity $\varphi_{0}$ :

$$
\frac{1}{M}=\frac{1}{N}+\frac{\varphi_{0}}{k_{f \ell}}
$$

By considering the pore solution as water, the fluid bulk modulus $k_{f \ell}$ can be estimated at $2.3 \mathrm{GPa}$. The total porosity of the cement paste $\varphi_{0}$ contains capillary pores and gel pores so that $\varphi_{0}=\varphi_{2}+f_{\mathrm{LD}} \phi_{\mathrm{LD}}+f_{\mathrm{HD}} \phi_{\mathrm{HD}}=f_{H}+f_{\mathrm{LD}} \phi_{\mathrm{LD}}+f_{\mathrm{HD}} \phi_{\mathrm{HD}}$ because the capillary pores remain saturated.

It is assumed that all the invariant elementary phases considered in this model and the resulting homogenized cement paste are isotropic. Then, the stiffness tensor $\mathbb{C}$ can be recast in the form $3 k \mathbb{J}+2 g \mathbb{K}$ where the fourth order tensors $\mathbb{J}$ and $\mathbb{K}$ stand for the spherical and deviatoric projections and are respectively equal to $1 / 31 \otimes \mathbf{1}$ and $\mathbb{I}-\mathbb{J}$. Likewise, the Biot tensor $\mathbf{B}$ is reformulated in $b \mathbf{1}$ where $b$ is referred to as the Biot-Willis parameter. The bulk and shear moduli, respectively $k$ and $g$, can be related to the elastic modulus $E$ and the Poisson's ratio $v$ with the following equations:

$$
E=\frac{9 k g}{3 k+g} ; \quad v=\frac{3 k-2 g}{6 k+2 g}
$$

The solid percolation threshold $t_{0}$ of the REV of cement paste is taken as the first instant at which a steep increase of the homogenized elastic modulus is observed.

\section{Polynomial chaos expansion and post-processing}

After having presented the poromechanics-hydration model, the randomness of the input parameters is assumed and the uncertainty of the poroelastic properties is apprehended by a spectral non-intrusive approach $[19,53]$. The model responses are represented by polynomial chaos expansions and the resulting metamodel is post-processed in order to extract statistical moments, perform a global sensitivity analysis and estimate PDF's for model outputs [54].

\subsection{Polynomial chaos representation}

The deterministic multiscale poromechanics-hydration model is noted $\mathcal{M}$. It has $M$ random input parameters $\left\{X_{i}, i=1, \ldots, M\right\}$ gathered in a vector $\underline{X}$ with prescribed probability density function (PDF) $f_{\underline{X}}(\underline{x})$ and predicts poroelastic properties at different time steps. All these predictions are represented by random variables gathered in $\left\{Y_{j}, j=1, \ldots, N\right\}$. This relation is expressed as follows:

$$
\underline{Y} \equiv \mathcal{M}(\underline{X})
$$

where $\underline{X}$ and $\underline{Y}$ are the random vectors of input parameters and model predictions. Their realizations are noted $\underline{x}$ and $y$. 


\subsubsection{Construction of the basis}

It is assumed that $\mathcal{M}$ is square integrable with respect to the probability measure $\mathbb{P}(d \underline{x})=f_{X}(\underline{x}) d \underline{x}$, meaning that every model response has a finite variance. Thereby, each output variable can be represented by a polynomial chaos expansion of the following form [19]:

$$
Y_{j}=\mathcal{M}^{(j)}(\underline{X}) \equiv \sum_{\underline{\alpha} \in \mathbb{N}^{M}} a_{\underline{\alpha}}^{(j)} \phi_{\underline{\alpha}}(\underline{X})
$$

where $\underline{\alpha}$ denotes all the possible multi-indices $\left(\alpha_{1}, \ldots, \alpha_{M}\right)$ with $\alpha_{i} \in \mathbb{N}, a_{\underline{\alpha}}^{(j)}$ refers to unknown deterministic coefficients and $\phi_{\underline{\alpha}}(\underline{x})$ to multivariate basis functions orthonormal with respect to the joint probability density function $f_{\underline{X}}(\underline{x})$ of the input parameters. The orthonormality is verified by the following inner product of the Hilbert space $\mathcal{H}=\mathcal{L}^{2}\left(\mathbb{R}^{M}, \mathbb{R}, \mathbb{P}(d \underline{x})\right)$ :

$$
\left\langle\phi_{\underline{\alpha}}(\underline{x}), \phi_{\underline{\beta}}(\underline{x})\right\rangle_{\mathcal{H}} \equiv \int_{\mathbb{R}^{M}} \phi_{\underline{\alpha}}(\underline{x}) \phi_{\underline{\beta}}(\underline{x}) f_{\underline{X}}(\underline{x}) \mathrm{d} \underline{x}=\delta_{\underline{\alpha} \underline{\beta}}
$$

where $\delta_{\underline{\alpha} \beta}=1$ if $\underline{\alpha}=\beta$ and 0 otherwise.

In the case of independent input parameters, the multivariate basis functions of Eq. (41) are expressed in terms of tensor products of univariate functions [54]:

$$
\phi_{\underline{\alpha}}(\underline{x}) \equiv \prod_{i=1}^{M} \pi_{\alpha_{i}}^{(i)}\left(x_{i}\right)
$$

Eq. (42) is verified by defining every univariate Hilbertian basis $\pi_{\alpha_{i}}^{(i)}\left(x_{i}\right)$ as the normalized function of a classical orthogonal polynomial. The choice of the suitable family of polynomials is dictated by the PDF of the input parameter $X_{i}$. In the sequel the input parameters of the multiscale poromechanics-hydration model are described either by $\operatorname{lognormal}(\ln \mathcal{N})$ or uniform $(\mathcal{U})$ distributions. In the former case, lognormal variables are transformed into Gaussian variables using the classical exponentiation and the associated Hermite polynomials (that are orthogonal with respect to the Gaussian measure) are used. In the latter case, so-called Legendre polynomials are used.

$$
\pi_{\alpha_{i}}^{(i)}\left(x_{i}\right)=\left\{\begin{aligned}
\left(2 \alpha_{i}+1\right)^{-1 / 2} L e_{\alpha_{i}}\left(x_{i}\right) & \text { if } X_{i} \sim \mathcal{U} \\
\left(\alpha_{i} !\right)^{-1 / 2} H e_{\alpha_{i}}\left(x_{i}\right) & \text { if } \ln X_{i} \sim \mathcal{N}
\end{aligned}\right.
$$

where $L e_{\alpha_{i}}$ and $H e_{\alpha_{i}}$ refer to the Legendre and Hermite polynomials of degree $\alpha_{i}$.

Thereby, the multiscale poromechanics-hydration model $\mathcal{M}(\underline{X})$ is replaced by a polynomial expansion of the form of Eq. (41). For practical application, one needs to determine the coefficients $a_{\underline{\alpha}}^{(j)}$ associated with each model prediction $Y_{j}$.

\subsubsection{Practical implementation}

For the sake of practical implementation, the infinite polynomial expansion of Eq. (41) is truncated and the model responses are approximated. This is done by limiting the expansion to multivariate polynomials of total degree less or equal to a fixed degree $p$. The total degree $q$ of any multivariate basis polynomial $\phi_{\underline{\alpha}}(\underline{x})$ is given by:

$$
q=|\underline{\alpha}| \equiv \sum_{i=1}^{M} \alpha_{i}
$$

The size $P$ of the corresponding finite set of coefficients $\left\{a_{\underline{\alpha}}^{(j)}, q=|\underline{\alpha}| \leq p, j=1, \ldots, N\right\}$ is:

$$
P=N\left(\begin{array}{c}
M+p \\
p
\end{array}\right)=N \frac{(M+p) !}{M ! p !}
$$

where $N$ is the number components of the model response.

Each model response can then be approximated by a serie of the following form:

$$
Y_{j}=\mathcal{M}^{(j)}(\underline{X}) \approx \sum_{|\underline{\alpha}| \leq p} a_{\underline{\alpha}}^{(j)} \phi_{\underline{\alpha}}(\underline{X})=\sum_{k=0}^{P / N-1} a_{k}^{(j)} \phi_{k}(\underline{X})
$$

The multi-indices $\underline{\alpha}$ of the last right-hand side are changed into $k$ indices in order to improve the understanding and simplify the forthcoming formulations. However, the amount of information contained by the multi-indices $\underline{\alpha}$ is greater than what the integer indices $k$ provide. Hence, a track is kept between the multi-indices $\underline{\alpha}$ and the coefficients $a_{k}^{(j)}$. Each coefficient $a_{k}^{(j)}$ is part of the following set:

$$
\mathcal{A}_{a}=\left\{a_{k}^{(j)}, k=0, \ldots, P / N-1, j=1, \ldots, N\right\}
$$

The coefficients of the truncated expansion can be determined by solving the following least-squares minimization problem:

$$
\mathcal{A}_{a}=\underset{a_{k}^{(j)} \in \mathbb{R}}{\arg \min } \sum_{j=1}^{N} \sum_{n=1}^{n_{s}}\left[\mathcal{M}^{(j)}\left(\underline{x}^{(n)}\right)-\sum_{k=0}^{P / N-1} a_{k}^{(j)} \phi_{k}\left(\underline{x}^{(n)}\right)\right]^{2}
$$

where $\underline{x}^{(n)}$ is a realization of the random input vector of parameters $\underline{X}$ among the experimental design $\mathcal{X}=\left\{\underline{x}^{(n)}, n=\right.$ $\left.1, \ldots, n_{s}\right\}$. The size of $\mathcal{X}$ needs to be at least as big as $P / N$ so that a solution exists for Eq. (49). It is usually recommended to take $\sim 2-3 P / N[53,55]$. The experimental design can be generated by Monte-Carlo simulation or Latin-Hypercube-Sampling (LHS).

\subsection{Post-processing}

Once the finite set of coefficients of Eq. (48) is correctly estimated, the truncated series expansion of Eq. (47) can be used as a surrogate of the multiscale poromechanics-hydration model in order to provide random predictions. Moreover, some quantitative information can be obtained by post-processing these coefficients without any need to simulate additional model responses. Indeed, the first statistical moments and Sobol' indices of the random outputs are straightforwardly computed that way. Eq. (47) can also be used together with Monte Carlo simulation in order to assess the PDF of the poroelastic properties by kernel smoothing techniques [56]. 


\subsubsection{First statistical moments}

The mean average value of every model prediction $\mathcal{M}^{(j)}(\underline{X})$ is the coefficient $a_{\underline{\alpha}}^{(j)}$ of order zero [54]:

$$
\mu_{Y_{j}} \equiv \mathbb{E}\left[Y_{j}\right]=a_{0}^{(j)}
$$

where it is assumed that the index $k$ equals 0 for $|\underline{\alpha}|=0$.

The variance of each model response can be obtained from the following expression:

$$
\sigma_{Y_{j}}^{2} \equiv \mathbb{V}\left[Y_{j}\right]=\sum_{i=1}^{P / N-1}\left(a_{i}^{(j)}\right)^{2}
$$

\subsubsection{Sobol' decomposition}

The univariate polynomials defined at Eq. (44) are independent on $x_{i}$ if $\alpha_{i}$ is equal to zero. Thereby, it is possible to identify the input parameters the multivariate polynomials are not functions of, and to reformulate the expansion of Eq. (41) by taking this information into account. An arbitrary set $\mathscr{I}_{i_{1} \ldots, i_{s}}$ that contains all the multi-indices $\underline{\alpha}$ for which $\left\{\alpha_{i}, i=i_{1}, \ldots, i_{s}\right\}$ are the only indices greater than zero is defined for this purpose:

$$
\mathscr{I}_{i_{1}, \ldots, i_{s}}=\left\{\underline{\alpha}: \begin{array}{c}
\alpha_{i}>0 \forall i=1, \ldots, M, \quad i \in\left(i_{1}, \ldots, i_{s}\right) \\
\alpha_{i}=0 \forall i=1, \ldots, M, \quad i \notin\left(i_{1}, \ldots, i_{s}\right)
\end{array}\right\}
$$

As presented by Sudret $[57,58]$, it is possible to gather the terms of Eq. (41) with respect to the input parameters they depend on:

$$
\begin{aligned}
\mathcal{M}^{(j)}(\underline{x})= & a_{0}^{(j)}+\sum_{i=1}^{M} \sum_{\underline{\alpha} \in \mathscr{I}_{i}} a_{\underline{\alpha}}^{(j)} \phi_{\underline{\underline{\alpha}}}\left(x_{i}\right) \\
& +\sum_{1 \leq i_{1}<i_{2} \leq M} \sum_{\underline{\alpha} \in \mathscr{I}_{i_{1}, i_{2}}} a_{\underline{\alpha}}^{(j)} \phi_{\underline{\alpha}}\left(x_{i_{1}}, x_{i_{2}}\right)+\ldots \\
& +\sum_{1 \leq i_{1}<\ldots<i_{s} \leq M} \sum_{\underline{\alpha} \in \mathscr{I}_{i_{1}, \ldots, i_{s}}} a_{\underline{\underline{\alpha}}}^{(j)} \phi_{\underline{\alpha}}\left(x_{i_{1}}, \ldots, x_{i_{s}}\right)+\ldots \\
& +\sum_{\underline{\alpha} \in \mathscr{I}_{1, \ldots, M}} a_{\underline{\alpha}}^{(j)} \phi_{\underline{\alpha}}\left(x_{1}, \ldots, x_{M}\right)
\end{aligned}
$$

where $a_{0}^{(j)}$ refers to the mean of $Y_{j}$ (see Eq. (50)). The summands of the above formulation constitute the unique Sobol' decomposition [59] of $Y_{j}$. Each summand can be expressed as follows [57]:

$$
\mathcal{M}_{i_{1}, \ldots, i_{s}}^{(j)}\left(x_{i_{1}}, \ldots, x_{i_{s}}\right)=\sum_{\underline{\alpha} \in \mathscr{I}_{i_{1}, \ldots, i_{s}}} a_{\underline{\alpha}}^{(j)} \phi_{\underline{\alpha}}\left(x_{i_{1}}, \ldots, x_{i_{s}}\right)
$$

where $\mathcal{M}_{i_{1}, \ldots, i_{s}}^{(j)}$ is the part of the model response $Y_{j}$ that depends only on the input parameters $\left\{x_{i}, i=i_{1}, \ldots, i_{s}\right\}$. Consequently, the part of the total variance that depends only on these parameters is:

$$
V_{i_{1}, \ldots, i_{s}}^{(j)}\left(x_{i_{1}}, \ldots, x_{i_{s}}\right)=\sum_{\underline{\alpha} \in \mathscr{I}_{i_{1}, \ldots, i_{s}}}\left(a_{\underline{\alpha}}^{(j)}\right)^{2}
$$

The relative contribution of any combination of random input parameters to the variance of $Y_{j}$ is straightforwardly expressed by the following polynomial chaos-based Sobol' indices [57]:

$$
S U_{i_{1}, \ldots, i_{s}}^{(j)}=V_{i_{1}, \ldots, i_{s}}^{(j)}\left(x_{i_{1}}, \ldots, x_{i_{s}}\right) / \sigma_{Y_{j}}^{2}
$$

where $\sigma_{Y_{j}}^{2}$ is given by Eq. (51) for a truncated polynomial expansion and a finite set of coefficients given by Eq. (48).

The Sobol' indices constitute the quantitative information used for the sensitivity analysis of model predictions to the uncertainty of the input parameters.

\subsubsection{Probability density function}

The polynomial chaos expansion in Eq. (47) may also be considered as a surrogate model of the original model $\mathcal{M}$. In order to estimate and plot the probability density function of any scalar random response a large sample set of points is drawn according to the input joint probability density function $f_{\underline{X}}(\underline{x})$, say $\left\{y_{j}^{(k)}, k=1, \ldots, n_{s}\right\}$ (e.g. $\left.n_{s}=10,000-100,000\right)$. Then the PDF of $\mathcal{M}^{(j)}(\underline{X})$ may be estimated by kernel smoothing:

$$
\hat{f}_{Y_{j}}\left(y_{j}\right)=\frac{1}{n_{s} h} \sum_{k=1}^{n_{s}} K\left(\frac{y_{j}-y_{j}^{(k)}}{h}\right)
$$

where $\hat{f}_{Y_{j}}\left(y_{j}\right)$ is the estimator of the PDF of $Y_{j}, n_{s}$ is the sample size, $K(\cdot)$ is a kernel function and $h$ is the bandwidth parameter. The kernel is a positive function defined such that $\int K\left(y_{j}\right) \mathrm{d} y_{j}=1$. The most usual kernels are Gaussian and Epanechnikov functions [56]. For a given kernel function, the quality of the estimation depends on the smoothness controlled by the band width parameter. Good results can be obtained from the empirical Silverman rule for the calculation of the bandwidth:

$$
h(K)=\left[\frac{8 \sqrt{\pi} R(K)}{3 \mu_{2}(K)^{2}}\right]^{1 / 5} \hat{\sigma} n_{s}^{-1 / 5}
$$

where $\hat{\sigma}$ is the estimator of the standard deviation of $Y_{j}$ based on the sample $\left\{y_{j}^{(k)}, k=1, \ldots, n_{s}\right\}$. This formulation is obtained by the minimization of the asymptotic mean integrated square error for densities not far from normal. $R(K)$ and $\mu_{2}(K)$ are obtained from:

$$
R(K)=\int K(y)^{2} \mathrm{~d} y ; \mu_{2}(K)=\int y^{2} K(y)^{2} \mathrm{~d} y
$$

\section{Model input parameters}

The multiscale poromechanics-hydration model presented by the authors requires the specification of four kinds of input parameters: the initial phase composition, the kinetic parameters of hydration, the invariant elastic properties and the morphological parameters of microstructure. Most of this information has either been directly characterized with uncertainty or assessed by inverse modeling and calibration. The aim of this section is to present the uncertainty considered for those input parameters. Therefore, each parameter is modeled as an independent variable which uncertainty is characterized by a given standard deviation and a model of probability distribution. Due to a lack of information available, no correlation is considered 
Table 2: Quantitative phase composition

\begin{tabular}{lcc}
\hline Anhydrous & \multicolumn{2}{l}{ Mass fraction, $m_{X}[1]$} \\
\cline { 2 - 3 } phase, $X$ & Mean & PDF \\
\hline $\mathrm{C}_{3} \mathrm{~S}$ & 0.622 & $\mathcal{U}(0.568,0.676)$ \\
$\mathrm{C}_{2} \mathrm{~S}$ & 0.152 & $\mathcal{U}(0.126,0.178)$ \\
$\mathrm{C}_{3} \mathrm{~A}$ & 0.106 & $\mathcal{U}(0.097,0.115)$ \\
$\mathrm{C}_{4} \mathrm{AF}$ & 0.009 & $\mathcal{U}(0.008,0.010)$ \\
$\mathrm{C}_{\overline{\mathrm{S}} \mathrm{H}_{2}}$ & 0.074 & $\mathcal{U}(0.068,0.080)$ \\
\hline
\end{tabular}

between the uncertain parameters. Although, if such information was available, some additional precautions such as the resort to Nataf transforms would be required [18]. The lack of experimental data also compels the authors to fix arbitrarily the coefficient of variation of some of the model parameters. Those choices may bias the results of sensitivity analysis; further research on the uncertainty of the input parameters is needed to assess this point.

\subsection{Phase composition}

The initial quantitative phase composition of the cement mixture (see Table 2) is determined by application of the Bogue method described in Section 2.

The inaccuracy of the mass fractions predicted by Bogue calculation has been discussed by several authors [23, 60-63]. The first reason for this discrepancy with the pure composition is that this method assumes that the clinker reaches equilibrium during cooling while it is very unlikely to happen in cement manufacture [23]. The non-consideration of the significant amounts of substitute ions present in the anhydrous phases and the attribution of the whole amount of major oxides (see Table 1) solely to the main clinker phases also contribute to estimation errors [23]. Without experimental data available, in this first approach, we reasonably assume those input parameters as uniform random variables. The mean of each mass fraction is taken equal to the estimation obtained from Section 2 and a coefficient of variation of $5 \%$ is considered. We consider only $5 \%$ of variation to preclude the emergence of a significant bias between the assumed uncertainty models and the realized distributions. Indeed, due to the constraint $m_{\mathrm{C}_{3} \mathrm{~S}}+m_{\mathrm{C}_{2} \mathrm{~S}}+m_{\mathrm{C}_{3} \mathrm{~A}}+m_{\mathrm{C}_{4} \mathrm{AF}}+m_{\mathrm{CS}_{\mathrm{H}}}=1$, every realization of $m_{X}$ is normalized by the sum of realizations $\sum_{X} m_{X}$. Such a normalization introduces some discrepancies between the assumed and realized dispersions of single mass fractions that turn to be negligible for coefficients of variation of $5 \%$.

\subsection{Kinetic parameters}

The input parameters of the hydration model described at Section 3.1 are summarized in Tables 3 and 4. The characteristic time $\tau_{X, 0}$, the reaction order $\kappa_{X}$, the diffusion coefficient $D_{X}$ and the hydration degrees $\xi_{X, 0}$ and $\xi_{X}^{*}$ that mark the advancement stages of hydration at transition between kinetic processes (see Section 3) are listed in Table 3 for $\mathrm{C}_{3} \mathrm{~S}, \mathrm{C}_{2} \mathrm{~S}, \mathrm{C}_{3} \mathrm{~A}$ and $\mathrm{C}_{4} \mathrm{AF}$. All those parameters were presented by Bernard et al. [6] and most of them vary as functions of water-to-cement ratio $(w / c)$. According to Berliner et al. [64], the reaction order $\kappa_{X}$ and the diffusion coefficient $D_{X}$ are considered as random input parameters while the characteristic time $\tau_{X, 0}$ and the hydration degrees are deterministic. The reaction orders are modeled by logarithmic PDF with coefficients of variation about $10 \%$ and the diffusion coefficients are also considered as lognormal random input parameters with coefficients of variation about $12 \%$.

The apparent activation energies presented in Table 4 are obtained from Bernard et al. [6]. Without precise quantitative information about their uncertainty, the authors consider these input parameters as uniform random variables with coefficients of variation of $5 \%$.

\subsection{Elastic parameters}

The input parameters of the multiscale poromechanics model presented at Section 3.2 are summarized in Table 5. Those are the elastic moduli $E$ and the Poisson's ratios $v$ of the elementary material phases presented in Fig. 2. Most of the elastic moduli have been determined from measurements obtained by nanoindentation while considering fixed values of Poisson's ratios. These Young moduli are defined as lognormal random input parameters with coefficients of variation between 5 and $20 \%$, depending on the values reported by the authors (see Table 5). A coefficient of variation of $10 \%$ is considered to fill the lack of quantitative information about uncertainty for gypsum, hydrogarnet, AFm and AFt. The Poisson's ratios are considered as deterministic input parameters for the following reasons: (i) Since most of the elastic moduli considered herein were obtained by means of nanoindentation assuming deterministic Poisson's ratios, it seems more consistent to the eyes of the authors to simulate the uncertainty of elastic moduli in the same conditions that the ones in which their variability was initially measured; (ii) It was demonstrated by Constantinides [10] that the effective elastic modulus of a REV homogenized with a self consistent scheme hardly depends on the Poisson's ratio of its solid inclusions; (iii) Sudret et al. [18] demonstrated by considering non-deterministic Poisson's ratios for the sensitivity analysis of a similar multiscale model that the homogenized elastic moduli are absolutely not sensitive to the uncertainty of the Poisson's ratios of the inclusions it contains.

\subsection{Microstructure parameters}

The morphological input parameters of microstructure are the aspect ratios $r_{X}$ of inclusions and the gel porosities $\phi_{X}$ of LD and HD C-S-H. Most of the inclusions of the multiscale model are considered spherical with a deterministic aspect ratio of 1 . Ettringite crystals are modeled by prolate ellipsoids with an aspect ratio $r_{\mathrm{AFt}}$ of 20 in order to take their sharpness into account. Portlandite inclusions are represented by oblate ellipsoids and their aspect ratio $r_{\mathrm{CH}}$ is set to 0.25 [37]. Both of these input parameters are defined as uniform random variables with a coefficient of variation of $10 \%$. The porosities $\phi_{\mathrm{LD}}$ and $\phi_{\mathrm{HD}}$ are respectively set to 37.3 and $24.7 \%$ with respect to the estimations of Jennings [31] and Ulm et al. [1]. Because the density of early-age calcium-silicate-hydrates is much less certain than 
Table 3: Kinetic parameters

\begin{tabular}{|c|c|c|c|c|c|c|c|c|c|c|}
\hline \multirow{2}{*}{$\begin{array}{c}\text { Phase, } \\
X\end{array}$} & \multirow{2}{*}{$\begin{array}{l}\mathrm{w} / \mathrm{c} \\
{[1]}\end{array}$} & \multirow{2}{*}{$\begin{array}{l}\tau_{X, 0} \\
{[\mathrm{~h}]}\end{array}$} & \multicolumn{3}{|l|}{$K_{X}[1]$} & \multirow{2}{*}{$\begin{array}{l}\xi_{X, 0} \\
{[1]}\end{array}$} & \multicolumn{3}{|l|}{$D_{X}\left[\mathrm{~cm}^{2} / \mathrm{h}\right]$} & \multirow{2}{*}{$\begin{array}{c}\xi_{X}^{*} \\
{[1]}\end{array}$} \\
\hline & & & Mean & SD & PDF & & Mean & SD & PDF & \\
\hline \multirow[t]{3}{*}{$\mathrm{C}_{3} \mathrm{~S}$} & 0.4 & 12.7 & 1.79 & 0.18 & $\ln \mathcal{N}$ & \multirow{3}{*}{0.02} & $1.05 \times 10^{-10}$ & $0.13 \times 10^{-10}$ & $\ln \mathcal{N}$ & \multirow{3}{*}{0.60} \\
\hline & 0.5 & 11.9 & 1.72 & 0.17 & $\ln \mathcal{N}$ & & $2.64 \times 10^{-10}$ & $0.32 \times 10^{-10}$ & $\ln \mathcal{N}$ & \\
\hline & 0.6 & 11.2 & 1.69 & 0.17 & $\ln \mathcal{N}$ & & $6.42 \times 10^{-10}$ & $0.77 \times 10^{-10}$ & $\ln \mathcal{N}$ & \\
\hline \multirow{3}{*}{$\mathrm{C}_{2} \mathrm{~S}$} & 0.4 & 66.1 & 1.03 & 0.10 & $\ln \mathcal{N}$ & \multirow{3}{*}{0.00} & \multirow{3}{*}{$6.64 \times 10^{-13}$} & \multirow{3}{*}{$0.80 \times 10^{-13}$} & \multirow{3}{*}{$\ln \mathcal{N}$} & \multirow{3}{*}{0.60} \\
\hline & 0.5 & 60.9 & 0.96 & 0.10 & $\ln \mathcal{N}$ & & & & & \\
\hline & 0.6 & 59.8 & 0.90 & 0.09 & $\ln \mathcal{N}$ & & & & & \\
\hline \multirow[t]{3}{*}{$\mathrm{C}_{3} \mathrm{~A}$} & 0.4 & 53.5 & 1.07 & 0.11 & $\ln \mathcal{N}$ & \multirow{3}{*}{0.04} & \multirow{3}{*}{$2.64 \times 10^{-10}$} & \multirow{3}{*}{$0.32 \times 10^{-10}$} & \multirow{3}{*}{$\ln \mathcal{N}$} & \multirow{3}{*}{0.60} \\
\hline & 0.5 & 49.2 & 1.00 & 0.10 & $\ln \mathcal{N}$ & & & & & \\
\hline & 0.6 & 42.6 & 0.93 & 0.09 & $\ln \mathcal{N}$ & & & & & \\
\hline \multirow[t]{3}{*}{$\mathrm{C}_{4} \mathrm{AF}$} & 0.4 & 24.2 & 2.37 & 0.24 & $\ln \mathcal{N}$ & \multirow{3}{*}{0.40} & $1.05 \times 10^{-10}$ & $0.13 \times 10^{-10}$ & $\ln \mathcal{N}$ & \multirow{3}{*}{0.60} \\
\hline & 0.5 & 21.4 & 2.30 & 0.23 & $\ln \mathcal{N}$ & & $2.64 \times 10^{-10}$ & $0.32 \times 10^{-10}$ & $\ln \mathcal{N}$ & \\
\hline & 0.6 & 17.9 & 2.23 & 0.22 & $\ln \mathcal{N}$ & & $6.42 \times 10^{-10}$ & $0.77 \times 10^{-10}$ & $\ln \mathcal{N}$ & \\
\hline
\end{tabular}

Table 4: Apparent activation energies

\begin{tabular}{lcc}
\hline Anhydrous & \multicolumn{2}{l}{$E_{a, X} / \mathscr{R}[\mathrm{K}]$} \\
\cline { 2 - 3 } phase, $X$ & Mean & PDF \\
\hline $\mathrm{C}_{3} \mathrm{~S}$ & 4500 & $\mathcal{U}(4110,4890)$ \\
$\mathrm{C}_{2} \mathrm{~S}$ & 2500 & $\mathcal{U}(2285,2715)$ \\
$\mathrm{C}_{3} \mathrm{~A}$ & 5500 & $\mathcal{U}(5025,5975)$ \\
$\mathrm{C}_{4} \mathrm{AF}$ & 4200 & $\mathcal{U}(3835,4565)$ \\
\hline
\end{tabular}

the density of latter hydration product [70], the porosity of LD $\mathrm{C}-\mathrm{S}-\mathrm{H}$ is considered as a random input parameter while $\phi_{\mathrm{HD}}$ is kept deterministic. A lognormal PDF is considered for $\phi_{\mathrm{LD}}$ with a coefficient of variation of $10 \%$.

\section{Results and discussion}

The multiscale poromechanics-hydration model proposed by the authors allows one to predict the solid percolation threshold of a cement paste as a function of the water-to-cement ratio for $0.35 \leq w / c \leq 0.70$. It also gives access to estimates of the BiotWillis parameter, the skeleton Biot modulus and the drained and undrained elastic moduli and Poisson's ratios as functions of time. The aim of this section is to validate this deterministic model, to present the results of uncertainty propagation from the input parameters, to identify the greatest contributors to the uncertainty of model predictions and to estimate the PDF of the drained elastic modulus at different time steps.

\subsection{Model validation}

The predictive capabilities of the deterministic model are evaluated with respect to the evolution of the undrained elastic modulus and Poisson's ratio compared to experimental data obtained by Boumiz et al. [20,21] on cement pastes similar to the materials considered in this study.
The evolution of the volume fractions required by the multiscale poromechanics model is presented in Fig. 2 for a water-tocement ratio of 0.50 at a curing temperature $T$ of $25^{\circ} \mathrm{C}$. The volume fractions of hydration products $\left(f_{\mathrm{LD}}, f_{\mathrm{HD}}, f_{\mathrm{CH}}, f_{\mathrm{AFt}}\right.$ and $\left.f_{\mathrm{C}_{3} \mathrm{AH}}\right)$ increase as a result of the decrease of the amounts of reactants $\left(f_{\mathrm{C}_{3} \mathrm{~s}}, f_{\mathrm{C}_{2} \mathrm{~s}}, f_{\mathrm{C}_{3} \mathrm{~A}}, f_{\mathrm{C}_{4} \mathrm{AF}}, f_{\mathrm{CS}_{2} \mathrm{H}_{2}}\right.$ and $\left.f_{\mathrm{H}}\right)$. The late reaction of ettringite to form AFm starts at an overall hydration degree $\xi$ of 0.68 and is not total; a remaining amount of $\mathrm{AFt}$ is predicted at the end of hydration.

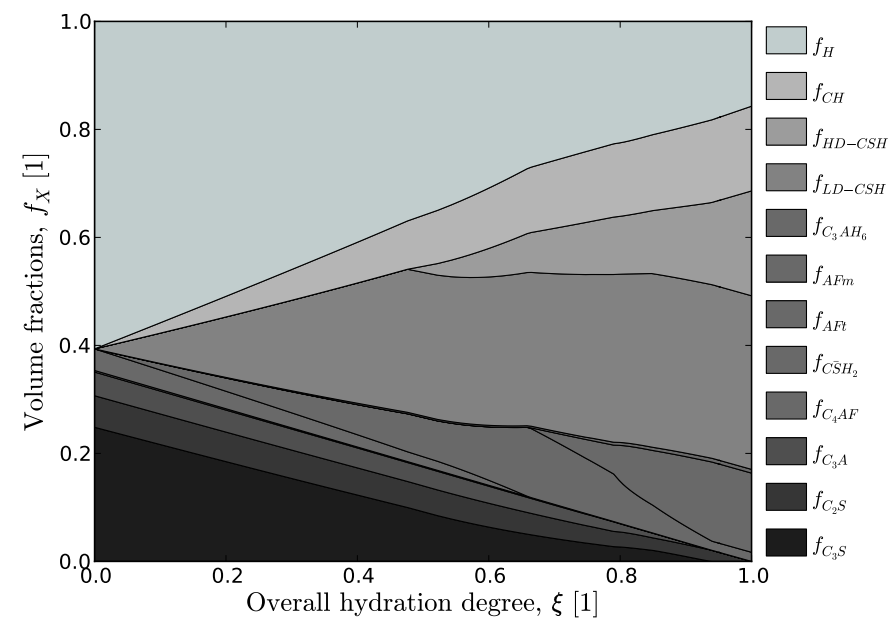

Figure 2:

The prediction of the undrained elastic modulus $E_{u}$ is presented in Fig. 3 as functions of the overall hydration degree and time. A good agreement is observed between those estimates and the experimental data of Boumiz et al. [20] obtained by acoustic wave measurements on saturated samples. The root mean square error computed between experiments and predictions are 1.22, 0.43 and $0.39 \mathrm{GPa}$ for water-to-cement ratios of $0.40,0.50$ and 0.60 . Graphically, the solid percolation 


\begin{tabular}{|c|c|c|c|c|c|c|}
\hline \multirow[t]{2}{*}{ Compound } & \multirow{2}{*}{$\begin{array}{c}\text { Nominal } \\
\text { formula }\end{array}$} & \multicolumn{3}{|c|}{$E[\mathrm{GPa}]$} & \multirow[t]{2}{*}{$v$} & \multirow[t]{2}{*}{ Ref. } \\
\hline & & Mean & SD & PDF & & \\
\hline $\begin{array}{l}\text { Tricalcium } \\
\text { silicate }\end{array}$ & $\mathrm{C}_{3} \mathrm{~S}$ & 135.0 & 7.0 & $\ln \mathcal{N}$ & 0.3 & Acker [65], Velez et al. [66] \\
\hline $\begin{array}{l}\text { Dicalcium } \\
\text { silicate }\end{array}$ & $\mathrm{C}_{2} \mathrm{~S}$ & 130.0 & 20.0 & $\ln \mathcal{N}$ & 0.3 & Acker [65], Velez et al. [66] \\
\hline $\begin{array}{l}\text { Tricalcium } \\
\text { aluminate }\end{array}$ & $\mathrm{C}_{3} \mathrm{~A}$ & 145.0 & 10.0 & $\ln \mathcal{N}$ & 0.3 & Acker [65], Velez et al. [66] \\
\hline $\begin{array}{l}\text { Tetracalcium } \\
\text { aluminoferrite }\end{array}$ & $\mathrm{C}_{4} \mathrm{AF}$ & 125.0 & 25.0 & $\ln \mathcal{N}$ & 0.3 & Acker [65], Velez et al. [66] \\
\hline Gypsum & $\mathrm{C} \overline{\mathrm{S}} \mathrm{H}_{2}$ & 45.7 & 4.6 & $\ln \mathcal{N}$ & 0.33 & $\begin{array}{l}\text { Choy et al. [67], } \\
\text { Bhalla et al. [68] }\end{array}$ \\
\hline Portlandite & $\mathrm{CH}$ & 38.0 & 5.0 & $\ln \mathcal{N}$ & 0.305 & Constantinides and Ulm [7] \\
\hline Hydrogarnet & $\mathrm{C}_{3}(\mathrm{~A}, \mathrm{~F}) \mathrm{H}_{6}$ & 22.4 & 2.2 & $\ln \mathcal{N}$ & 0.25 & Kamali et al. [69] \\
\hline AFm & $\mathrm{C}_{4} \mathrm{~A} \overline{\mathrm{S}} \mathrm{H}_{12}$ & 42.3 & 4.2 & $\ln \mathcal{N}$ & 0.324 & Kamali et al. [69] \\
\hline $\mathrm{AFt}$ & $\mathrm{C}_{6} \mathrm{~A}_{\overline{\mathrm{S}}} \mathrm{H}_{32}$ & 22.4 & 2.2 & $\ln \mathcal{N}$ & 0.25 & Kamali et al. [69] \\
\hline LD C-S-H & $\mathrm{C}_{3.4} \mathrm{~S}_{2} \mathrm{H}_{8}$ & 21.7 & 2.2 & $\ln \mathcal{N}$ & 0.24 & Constantinides and Ulm [7] \\
\hline HD C-S-H & $\mathrm{C}_{3.4} \mathrm{~S}_{2} \mathrm{H}_{8}$ & 29.4 & 2.4 & $\ln \mathcal{N}$ & 0.24 & Constantinides and Ulm [7] \\
\hline
\end{tabular}

thresholds can be interpreted as the times $t_{0}$ or hydration degrees where the curves of undrained elastic moduli intercept the horizontal axes. The greatest discrepancy with experimental data is observed for the mix of water-to-cement ratio of 0.40 .

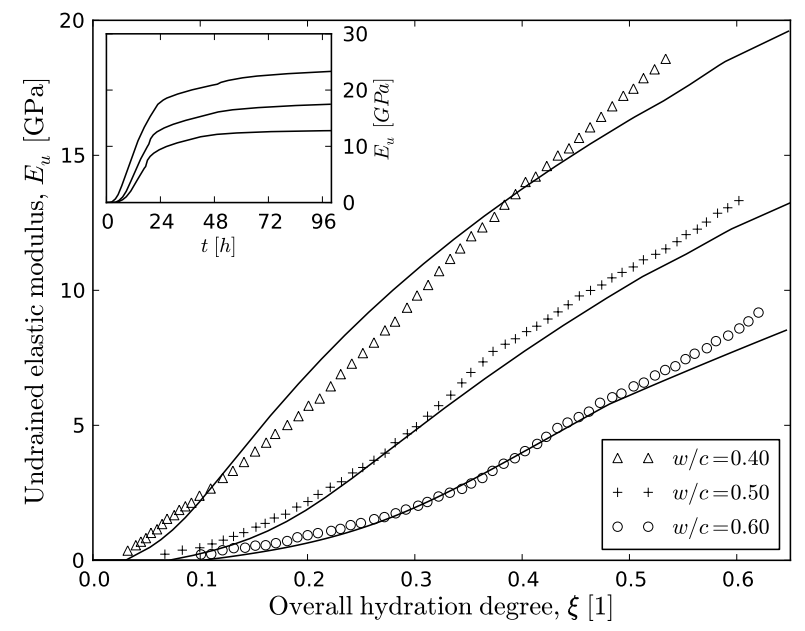

Figure 3:

The predictions of the Poisson's ratios for the three mix designs of the study are presented in Fig. 4 and compared to experimental data from Boumiz et al. [21] at a water-to-cement ratio of 0.40 . The model predicts a faster decrease of the Poisson's ratio than the experimental data. However, the steady value reached after the hydration of $50 \%$ of the cement suffers less from discrepancy with observations than the shape of the predicted relation $v_{u}(t)$. We remind here that none of the studies conducted by Bernard et al. [6], Sanahuja et al. [15] and Pichler et al. $[39,51]$ presented a comparison of their predictions of the
Poisson's ratio with experimental data.

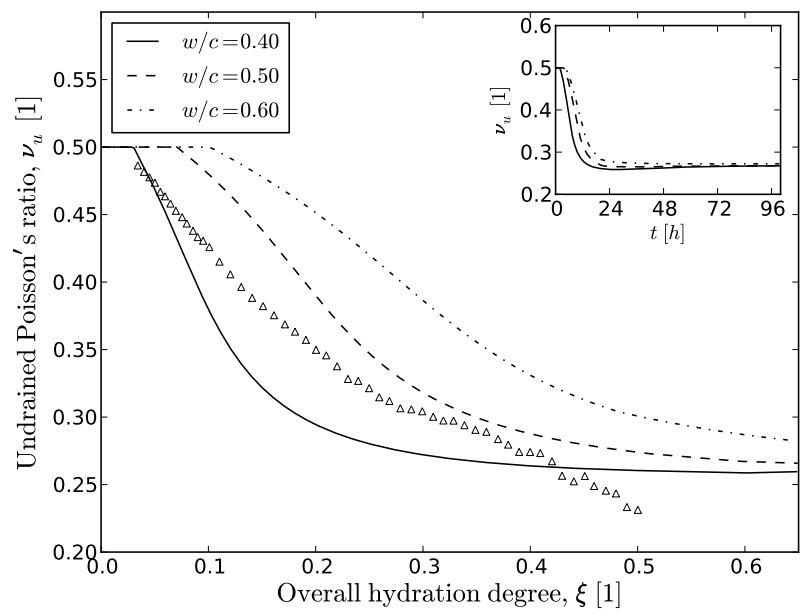

Figure 4:

The multiscale poromechanics-hydration model is validated for its capaciy to predict the evolution at early-age of the elastic properties of cement pastes with water-to-cement ratios between 0.35 and 0.70 . The upper-limit value of $w / c$ is imposed by the validity domain of the kinetic parameters calibrated by Berliner et al. [64] and the lower value is prescribed by the limitation of the self-consistent homogenization scheme adopted at level II (see Section 3.2.2) to model percolation. Indeed, the multiscale poromechanics-hydration model predicts initial stiffnesses significantly greater than zero for mix designs of waterto-cement ratios lower than 0.35 . 


\subsection{Uncertainty propagation}

A set of $n_{t}$ deterministic time steps is prescribed for the three mix designs of this study $(w / c=0.40,0.50,0.60)$. For each mix, the solid percolation threshold $t_{0}$ is computed and the drained elastic modulus $E$, the Biot-Willis parameter $b$ and the skeleton Biot modulus $N$ are simulated at every time step. There are $3 n_{t}+1$ model responses calculated by mix and 31 input parameters. A degree $p=2$ is prescribed for the truncature of the polynomial expansion (see Eq. (47)) so that the number of unknown coefficients is 528 per model response. According to precedent works $[53,71,72]$, these coefficients can be accurately determined by regression from experimental designs of sizes 2 to 3 times greater than the number of unknowns. An experimental design that contains 2,000 realizations by model response is drawn by LHS. The calculations are carried out using the open source software for uncertainty quantification Open TURNS (http://www.openturns.org/).

Means and variances are computed with respect to Eqs. (50) and (51). The evolution of the means plus or minus a standard deviation and the coefficients of variation of the poroelastic properties $(E, b, N)$ are represented as functions of time in Fig. 5. The uncertainties of the model predictions are described as follows:

Drained elastic modulus, $E$ : A very high uncertainty is observed during the first 12 hours, when percolation is susceptible to happen. The lower the water-to-cement ratio, the faster is the decrease of the coefficient of variation towards a limit of $5 \%$ independent of the mix design. In comparison to the uncertainty models of input parameters (see Section 5), the propagation of randomness through scales up to the macroscopic elastic modulus tends to diminish the uncertainty.

Biot-Willis parameter, $b$ : The initial uncertainty is negligible but quickly increases during the first 12 hours of hydration. The limit coefficients of variation lie between 1.5 and $4 \%$ and are inversely proportional to the water-to-cement ratio. The propagation through scales does not show any magnification of the uncertainty.

Skeleton Biot modulus, $N$ : The coefficients of variation of the skeleton Biot modulus vary between 5 and $10 \%$. The uncertainty of the mix design with a water-to-cement ratio of 0.40 exhibits a significant increase during the first 24 hours.

Solid percolation threshold, $t_{0}$ : The mean predictions of the solid percolation threshold are 1.01, 2.06 and $2.60 \mathrm{~h}$ respectively for water-to-cement ratios of $0.40,0.50$ and 0.60 . The coefficients of variation are $2.5,6.3$ and $9.6 \%$. The greater the initial amount of water, the greater is the uncertainty on setting predictions.

Fig. 5 also presents the evolution of pairwise correlations among the macroscopic poroelastic properties as a function of time. The elastic modulus $E$ and the Biot-Willis parameter $b$ are negatively correlated. According to the counteracting contribution of pore pressure and strain in the first poromechanics state equation (see Eq. 29), this correlation is likely to amplify the uncertainty of the computed stress acting over an isotropic element of cement paste subjected to tensile strains. In the case of a prescribed pore pressure with stress-free deformations (e.g. autogenous shrinkage), this correlation may be responsible of a magnification of the uncertainty of the computed volume changes. Similarly, beyond 12 hours of hydration, the joint uncertainty of the skeleton Biot modulus $N$ and the Biot-Willis parameter $b$ amplifies the uncertainty of the porosity change predicted by Eq. (33).

The pairwise correlations of the poroelastic properties with the solid percolation threshold are presented in Fig. 6 as a function of time. The more the hydration process is advanced, the less significant is the correlation between the setting time and those macroscopic properties. This is consistent with the fact that different micromechanics-hydration models [6, 15, 39] can predict significantly different behaviors during the first hours of hydration while providing equally good estimates of mature elastic properties. The correlations of the Biot-Willis parameter tensor and the skeleton Biot modulus with $t_{0}$ decrease faster than $\rho_{E, t_{0}}(t)$.

\subsection{Global sensitivity analysis}

The results of the global sensitivity analysis are respectively presented in Figs. 7, 8 and 9 for the percolation threshold, the drained elastic modulus and the Biot-Willis parameter. For the sake of clarity, the 31 input parameters were gathered into four categories (see Section 5) and allocated as follows: 5 initial amounts of anhydrous cement phases, 12 kinetic parameters, 11 elastic moduli and 3 morphological parameters of microstructure.

The contribution of each category consists in the sum of the first order Sobol' indices (see Eq. (57)) of the input parameters belonging to the category. The total sum of these indices is almost 1 for every model response. This means that there are negligible interaction effects between these variables. Hence, only the univariate contributions are considered in the sequel.

The sensitivity of model predictions to the uncertainty of model parameters is as follows:

Solid percolation threshold, $t_{0}$ : Since the work of Sanahuja et al. [15], it is clear that the solid percolation threshold of cement paste predicted by a micromechanics-based approach is closely related to the morphology of solid inclusions. Therefore, we expect the uncertainty of the aspect ratio of ettringite and portlandite to have a significant influence on the accuracy of the predicted percolation threshold. We also remind that for a given aspect ratio of solid particles at level II, percolation occurs at a critical porosity under which the REV stiffens for a growing packing density. The critical porosity is independent of the mechanical properties of solid phases as it solely depends on the aspect ratio of particles [15]. In other words, while a stiffer hydration product precipitated at early-age is expected to increase the stiffening rate of the paste, it is not expected to hasten percolation. Therefore, we also expect the predicted percolation threshold to be sensitive to the uncertain parameters that are mostly responsible for the decrease of porosity at earlyage. Now, we know from the results of sensitivity analysis that the greatest contributors to the uncertainty of the percolation threshold are the kinetic parameters. Independently of any category, the variability of the apparent activation energy of aluminate $E_{a, \mathrm{C}_{3} \mathrm{~A}} / \mathscr{R}$, of the aspect ratio of ettringite $r_{\mathrm{AFt}}$ and of the as- 


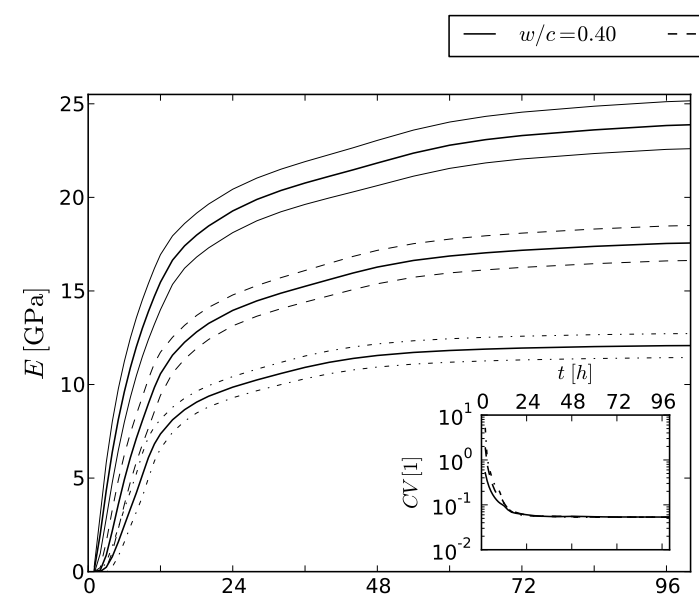

$$
w / c=0.50 \quad \cdots \quad w / c=0.60
$$
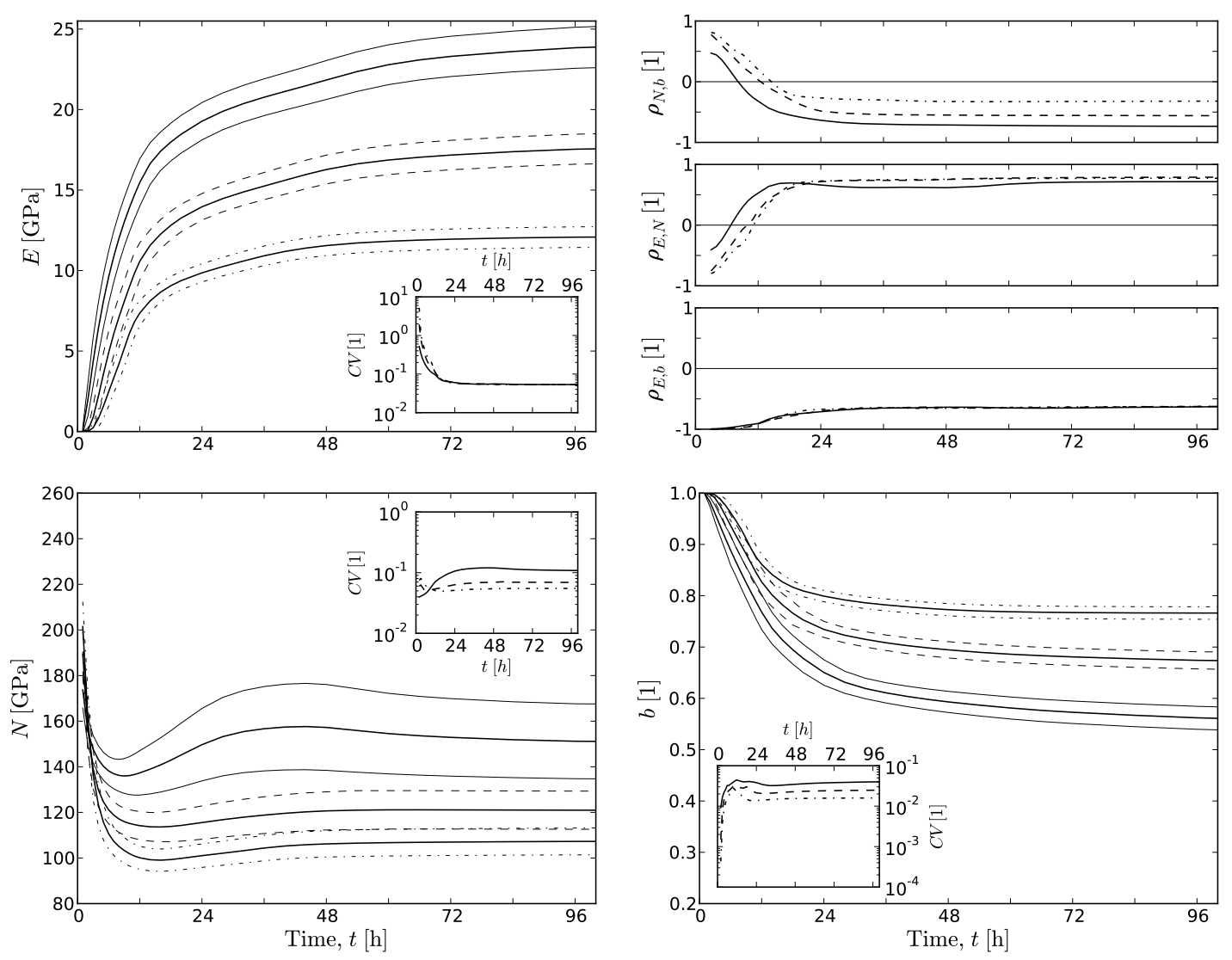

Figure 5:

pect ratio of portlandite $r_{\mathrm{CH}}$ is responsible for more than $94 \%$ of the uncertainty of the setting time for any mix design. However, the total contribution of the microstructure parameters does not go over $19.6 \%$. The quantitative phase composition has a negligible influence and the elastic moduli of the elementary material phases do not contribute at all. Those results are not surprising since the hydration of aluminate consumes significantly more water that any other reactant at early-age (see Eq. (3)). Now, before percolation, all the water consumed by hydration involves a decrease of porosity at level II so that the REV gets closer to the configuration described by its critical porosity. We also note that all the activation energies are negatively correlated with the percolation threshold, which is consistent with respect to the functional relationship between the reaction rate and activation energy at early-age (see Eq. (7)). Finally, the results of the sensitivity analysis reveal that the lower the waterto-cement ratio, the lower is the influence of the kinetic parameters and the bigger is the contribution of the shape of inclusions.

Drained elastic modulus, E: The most important contribution to the variability of the elastic modulus during the first 12 hours (see Fig. 8) comes from the kinetic parameters and, more precisely, the apparent activation energy of aluminate. The part of variance due to the elastic moduli of the elementary material phases increases with time until the end of hydration. After 18 hours, the elastic parameters govern and $E_{\mathrm{LD}}$ is the greatest source of uncertainty. After 36 hours, the second greatest contributor is the elastic modulus of portlandite, and at $96 \mathrm{~h}$, approximately $60 \%$ of the variance is due to $E_{\mathrm{LD}}$. The microstructure parameters and the quantitative phase composition have negligible effects. The sensitivity of the elastic modulus of cement paste does not vary much as a function of the waterto-cement ratio. Those results are not surprising since, as it was pointed out by Sanahuja et al. [15], the smaller the porosity, the smaller the dependence of the effective elastic properties on the morphology of solid particles. Moreover, the significant contribution of uncertainty of the elastic moduli of $\mathrm{C}-\mathrm{S}-\mathrm{H}$ and portlandite is due to the fact that they are the hydration products present in largest quantities after ettringite starts to dissolve (see Fig. 2 for $w / c=0.50)$.

Biot-Willis parameter, $b$ : The Biot-Willis parameter $b$ exhibits the same decreasing sensitivity to the apparent activation energy $E_{a, \mathrm{C}_{3} \mathrm{~A}} / \mathscr{R}$ (Fig. 9). However, the rate of the increasing part of variance due to the elastic moduli is slower. After 12 hours, the quantitative phase composition starts to influence the 

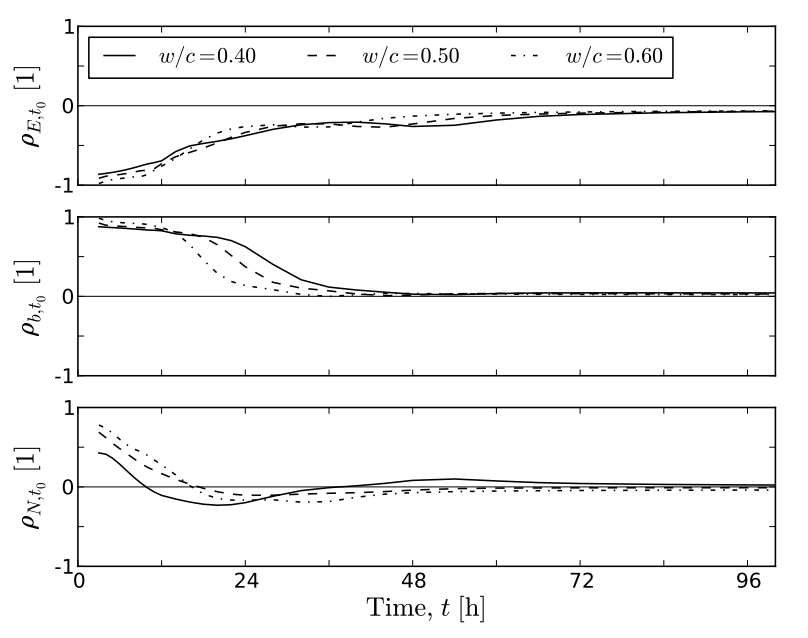

Figure 6:

uncertainty and this contribution grows until it lies between 14 and $22 \%$, depending on the water-to-cement ratio.

\subsection{Probability density function}

The stochastic metamodel (see Eq. (47)) is used to generate samples of 7,000 realizations of the elastic modulus at different time steps. The PDF of these response quantities is estimated by Gaussian kernel smoothing (see Section 4.2.3) and represented in Fig. 10. Then, extreme values of the elastic modulus can be obtained from those distributions. For instance, the $5 \%$ quantile is represented in Fig. 10.

Before using such PDFs for consistent reliability analysis, it could be relevant to confront the uncertainty propagation predicted in this study to experimental observations of the propagation of randomness through scales. A part of this information could be obtained by an extensive application of nondestructive mechanical testing procedures at nano- and macroscale.

\section{Conclusion}

A multiscale poromechanics-hydration model was proposed to estimate the Biot-Willis parameter, the skeleton Biot modulus and the drained and undrained elastic moduli and Poisson's ratio of hydrating cement paste as functions of time. The shapes of the inclusions of ettringite and portlandite were considered for a better prediction of elastic properties during the first hours of hydration compared to the results obtained by Bernard et al. [6]. These properties can then be used in macroscopic state equations to model the poromechanical behavior of partially saturated cement paste. The model was developed for a range of water-to-cement ratio between 0.35 and 0.70 . It was herein validated for water-to-cement ratios of $0.40,0.50$ and 0.60 . We remind that the model is based on the assumption that the physics of solid percolation in cement paste is adequately taken into account through the self consistent homogenization scheme. However, the fact that it predicts percolation even before the start of hydration for water-to-cement ratios under the lower limit of 0.35 reveals a limitation of the micromechanicsbased approach to model percolation in cement-based materials.

We employed a powerful probabilistic approach based on polynomial chaos expansion to propagate the uncertainty of the phase composition, the kinetic parameters of hydration, the elastic moduli and the morphological parameters through length scales. The polynomial expansion allows to predict variability and identify the greatest contributors to this variability among the uncertain input parameters. Although the results may be biased by some arbitrary choices of uncertainty model, they show that: the propagation does not magnify the uncertainty for the single poroelastic properties although, their correlation may amplify the variability of the estimates obtained from the macroscopic state equations. In order to reduce the uncertainty of the percolation threshold and the poroelastic properties at early-age, engineers should attempt to decrease the uncertainty of the apparent activation energy of calcium aluminate. Later on, the variability of the poroelastic properties can be reduced by improving the accuracy of the elastic modulus of LD C-S-H. This work is a first step for us towards a probabilistic microporomechanics approach for assessing volume changes at early-age and risk of cracking directly from relevant features of the microstructure of cement-based materials.

\section{Acknowledgements}

The first author wishes to acknowledge the financial participation of the Natural Sciences and Engineering Research Council of Canada (NSERC), the Québec Fund for Nature and Technology Research (FQRNT), the Research Center on Concrete Infrastructures (CRIB) and Phimeca Engineering. The authors also thank Yann Caniou for his helpful advices about the open source software Open TURNS (http://www.openturns.org/).

\section{List of Figures}

1 Multiscale representation of the microstructure of cement paste, adapted from Constantinides [5]. 4

2 Model predictions of the volume fractions for $\mathrm{w} / \mathrm{c}=0.50 \ldots \ldots \ldots \ldots \ldots \ldots \ldots$

3 Model predictions of the undrained elastic modulus and experimental data from Boumiz et al. [20].. . . . . . . . . . . . . .

4 Model predictions of the undrained Poisson's ratio and experimental data for $\mathrm{w} / \mathrm{c}=0.40$ from Boumiz et al. [21]. . . . . . . . . . . . . .

5 Prediction of the uncertainty of poroelastic properties of cement paste and pairwise correlations . . . . . . . . . . . . . .

6 Pairwise correlations of the poroelastic properties with the percolation threshold. . . . . . . . 15

7 Sensitivity analysis of the percolation threshold - First Sobol' indices. . . . . . . . . . . . . . . 16 


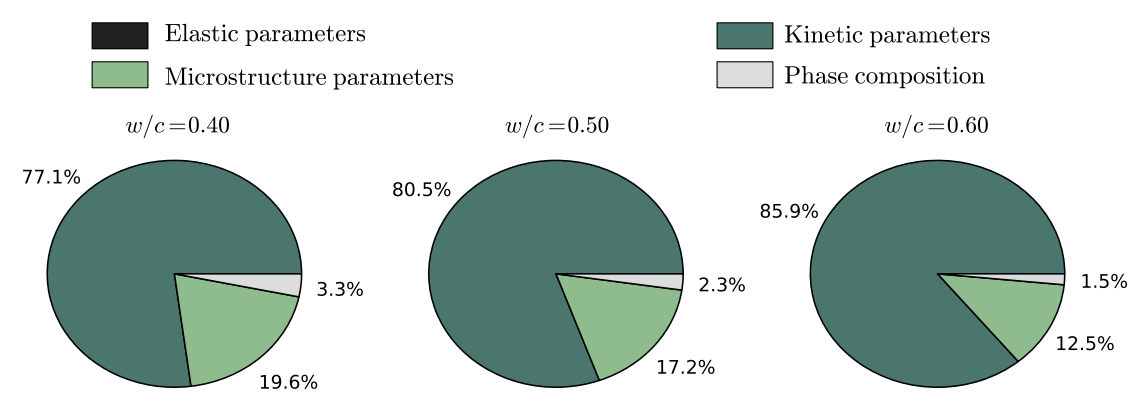

Figure 7:

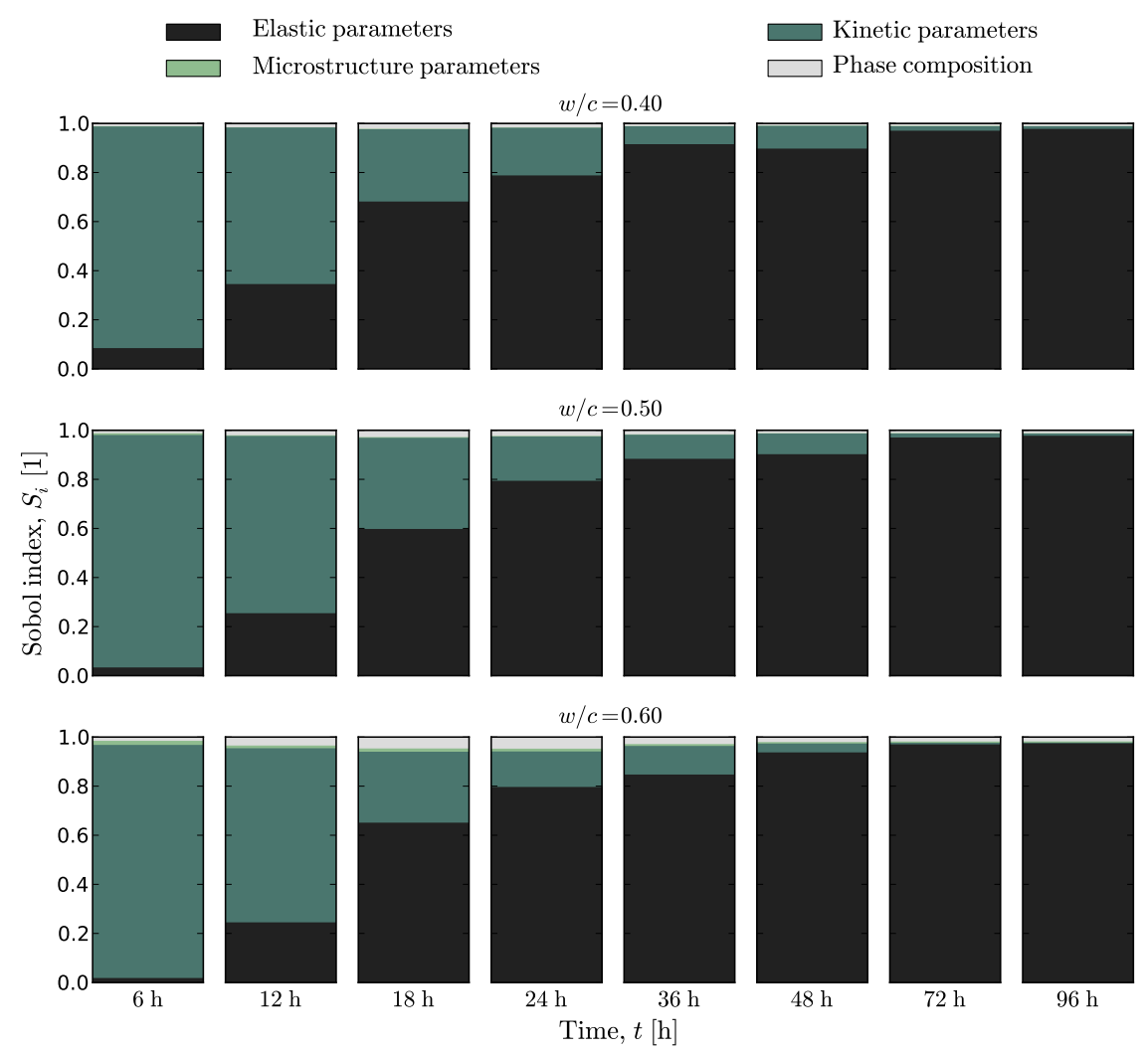

Figure 8: 


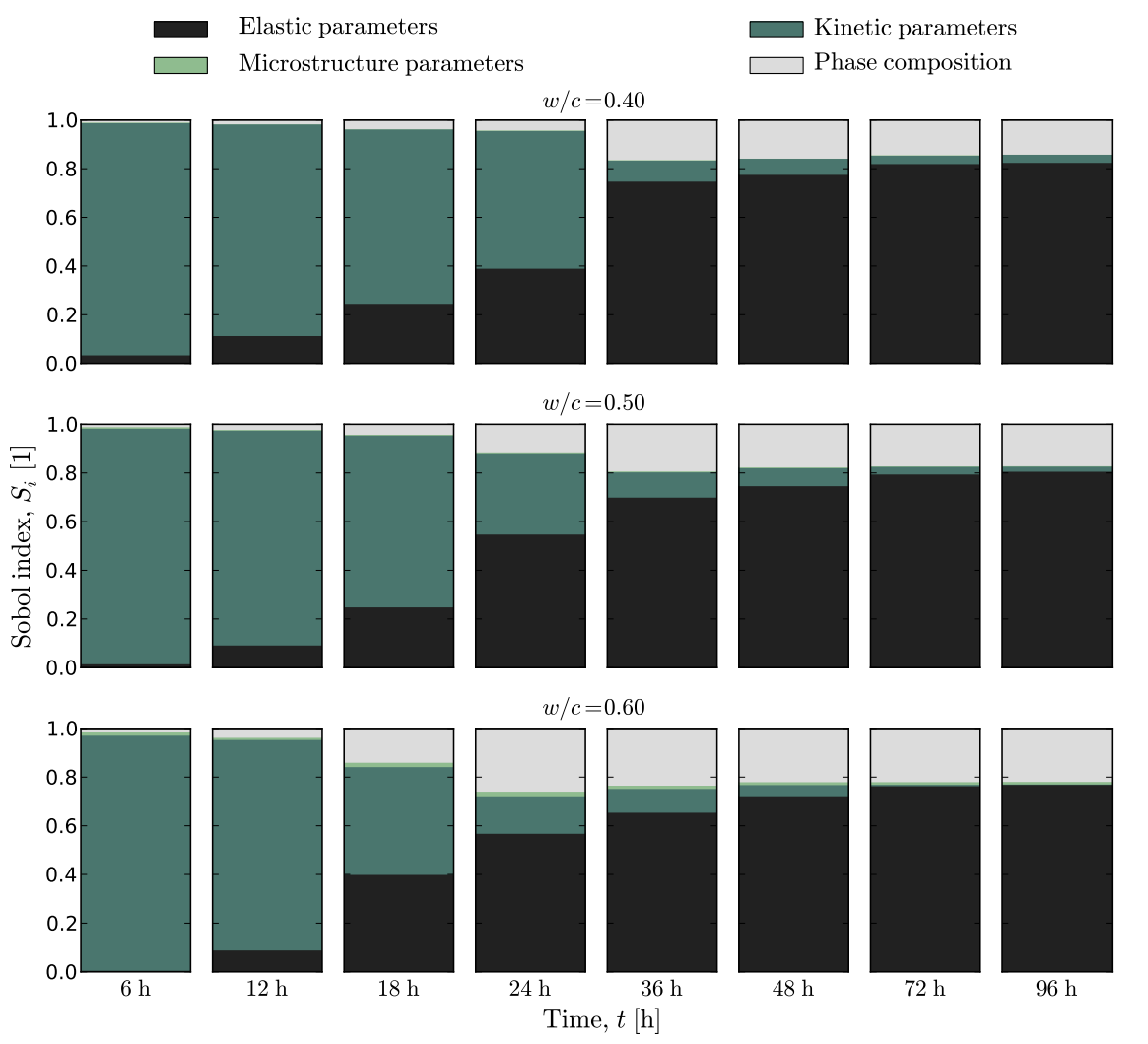

Figure 9:

8 Sensitivity analysis of the drained elastic moduls - First order Sobol' indices. . . . . . . 16

9 Sensitivity analysis of the Biot-Willis parameter - First order Sobol' indices. . . . . . . . . 17

10 Smoothed probability density function of the drained elastic modulus as a function of time. . 17

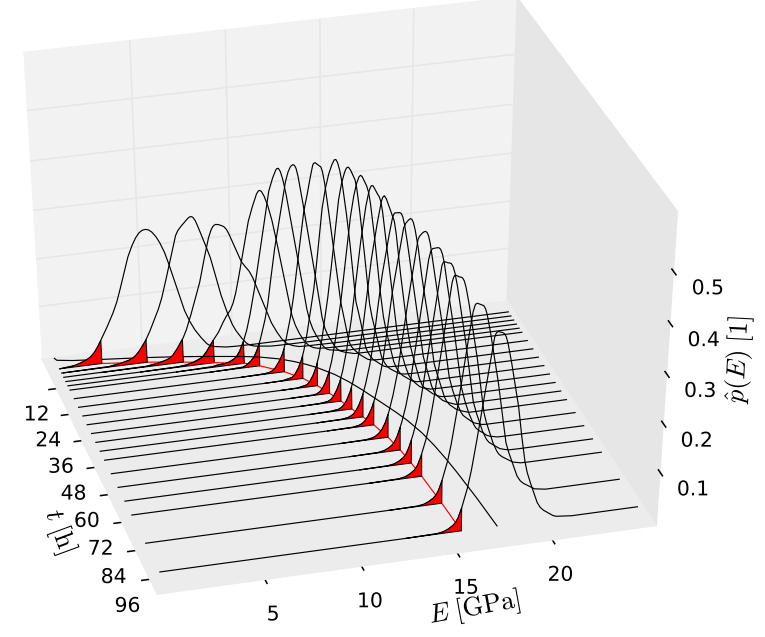

Figure 10:

\section{References}

[1] F. Ulm, G. Constantinides, F. Heukamp, Is concrete a poromechanics materials? - A multiscale investigation of poroelastic properties, Materials and Structures 37 (1) (2004) 43-58.

[2] B. Pichler, S. Cariou, L. Dormieux, Damage evolution in an underground gallery induced by drying, International Journal For Multiscale Computational Engineering 7 (2) (2009) 65-89.

[3] B. Pichler, L. Dormieux, Cracking risk of partially saturated porous media-Part I: Microporoelasticity model, Int. J. Numer. Anal. Meth. Geomech. 34 (2) (2010) 135-157.

[4] B. Pichler, L. Dormieux, Cracking risk of partially saturated porous media-Part II: Application to drying shrinkage, Int. J. Numer. Anal. Meth. Geomech. 34 (2) (2010) 159-186.

[5] G. Constantinides, The elastic properties of calcium leached cement pastes and mortars: A multi-scale investigation, Master's thesis, Massachusetts Institute of Technology (2002).

[6] O. Bernard, F.-J. Ulm, E. Lemarchand, A multiscale micromechanicshydration model for the early-age elastic properties of cement-based materials, Cement and Concrete Research 33 (9) (2003) 1293-1309.

[7] G. Constantinides, F.-J. Ulm, The effect of two types of C-S-H on the elasticity of cement-based materials: Results from nanoindentation and micromechanical modeling, Cement and Concrete Research 34 (1) (2004) 67-80. 
[8] L. Sorelli, G. Constantinides, F.-J. Ulm, F. Toutlemonde, The nanomechanical signature of ultra high performance concrete by statistical nanoindentation techniques, Cement and Concrete Research 38 (12) (2008) 1447-1456.

[9] L. Dormieux, E. Lemarchand, D. Kondo, E. Fairbairn, Elements of poromicromechanics applied to concrete, Materials and Structures 37 (1) (2004) 31-42.

[10] G. Constantinides, Invariant mechanical properties of calcium-silicatehydrates, Ph.D. thesis, Massachusetts Institute of Technology (2006).

[11] C. Pichler, R. Lackner, H. A. Mang, A multiscale micromechanics model for the autogenous-shrinkage deformation of early-age cement-based materials, Engineering Fracture Mechanics 74 (1-2) (2007) 34-58.

[12] F. Lin, C. Meyer, Modeling shrinkage of portland cement paste, ACI Materials Journal 105 (3) (2008) 302-311.

[13] F. Grondin, M. Bouasker, P. Mounanga, A. Khelidj, A. Perronnet, Physico-chemical deformations of solidifying cementitious systems: multiscale modelling, Materials and Structures 43 (1) (2010) 151-165.

[14] L. Stefan, F. Benboudjema, J. M. Torrenti, B. Bissonnette, Prediction of elastic properties of cement pastes at early ages, Computational Materials Science 47 (3) (2010) 775-784.

[15] J. Sanahuja, L. Dormieux, G. Chanvillard, Modelling elasticity of a hydrating cement paste, Cement and Concrete Research 37 (10) (2007) 1427-1439.

[16] J. Sanahuja, Impact de la morphologie structurale sur les performances mécaniques des matériaux de construction: Application au plâtre et à la pâte de ciment, Ph.D. thesis, École Nationale des Ponts et Chaussées (2008).

[17] M. Berveiller, Y. Le Pape, J. Sanahuja, A. Giorla, Sensitivity analysis and uncertainty propagation in multiscale modeling of concrete, 4th Biot Conference on Poromechanics (2009).

[18] B. Sudret, T. Yalamas, E. Noret, P. Willaume, Sensitivity analysis of nested multiphysics models using polynomial chaos expansions, Safety, Reliability and Risk of Structures, Infrastructures and Engineering Systems - Furuta, Frangopol \& Shinozuka (eds) (2010) 3883-3890.

[19] C. Soize, R. Ghanem, Physical systems with random uncertainties: Chaos representations with arbitrary probability measure, SIAM J. Sci. Comput. 26 (2) (2004) 395-410.

[20] A. Boumiz, C. Vernet, F. Tenoudji, Mechanical properties of cement pastes and mortars at early ages: Evolution with time and degree of hydration, Advanced Cement Based Materials 3 (3-4) (1996) 94-106.

[21] A. Boumiz, D. Sorrentino, C. Vernet, F. C. Tenoudji, Modelling the development of the elastic moduli as a function of the hydration degree of cement pastes and mortars, 2nd International Rilem Workshop On Hydration and Setting: Why Does Cement Set? An Interdisciplinary Approach 13 (2000) 295-316.

[22] R. H. Bogue, Calculation of the compounds in portland cement, Industrial \& Engineering Chemistry Analytical Edition 14 (1929) 192-197.

[23] H. Taylor, Cement chemistry, Academic Press, 1990.

[24] T. Powers, T. Brownyard, Studies of the physical properties of hardened portland cement paste (nine parts), Journal of the American Concrete Institute 43 (Oct. 1946 to April 1947) 101-992.

[25] P. D. Tennis, H. M. Jennings, A model for two types of calcium silicate hydrate in the microstructure of portland cement pastes, Cement and Concrete Research 30 (6) (2000) 855-863.

[26] M. Avrami, Kinetics of phase change. I, Journal of Chemical Physics 7 (1939) 1103-1112.

[27] K. Fujii, W. Kondo, Kinetics of the hydration of tricalcium silicate, Journal of the American Ceramic Society 57 (11) (1974) 492-497.

[28] L. Buffo-Lacarrière, A. Sellier, G. Escadeillas, A. Turatsinze, Multiphasic finite element modeling of concrete hydration, Cement and Concrete Research 37 (2) (2007) 131-138.

[29] E. Villa, P. R. Rios, Transformation kinetics for surface and bulk nucleation, Acta Materialia 58 (7) (2010) 2752-2768.

[30] G. W. Scherer, Models of confined growth, Cement and Concrete Research 42 (9) (2012) 1252-1260.

[31] H. M. Jennings, A model for the microstructure of calcium silicate hydrate in cement paste, Cement and Concrete Research 30 (1) (2000) 101116.

[32] J. Sanahuja, L. Dormieux, G. Chanvillard, A reply to the discussion "does C-S-H particle shape matter?" F.-J. Ulm and H. Jennings of the paper "modelling elasticity of a hydrating cement paste", CCR 37 (2007), Ce- ment and Concrete Research 38 (8-9) (2008) 1130-1134.

[33] H. M. Jennings, B. J. Dalgeish, P. L. Pratt, Morphological development of hydrating tricalcium silicate as examined by electron-microscopy techniques, Journal of the American Ceramic Society 64 (10) (1981) 567-572.

[34] P. Fonseca, H. Jennings, The effect of drying on early-age morphology of $\mathrm{C}-\mathrm{S}-\mathrm{H}$ as observed in environmental SEM, Cement and Concrete Research 40 (12) (2010) 1673-1680.

[35] A. Zaoui, Continuum micromechanics: Survey, J. Eng. Mech. 128 (8) (2002) 808-816.

[36] H. M. Jennings, Refinements to colloid model of C-S-H in cement: CMII, Cement and Concrete Research 38 (3) (2008) 275-289.

[37] E. Stora, Q.-C. He, B. Bary, Influence of inclusion shapes on the effective linear elastic properties of hardened cement pastes, Cement and Concrete Research 36 (7) (2006) 1330-1344.

[38] B. Pichler, S. Scheiner, C. Hellmich, From micron-sized needle-shaped hydrates to meter-sized shotcrete tunnel shells: micromechanical upscaling of stiffness and strength of hydrating shotcrete, Acta Geotechnica 3 (4) (2008) 273-294.

[39] B. Pichler, C. Hellmich, J. Eberhardsteiner, Spherical and acicular representation of hydrates in a micromechanical model for cement paste: prediction of early-age elasticity and strength, Acta Mechanica 203 (3-4) (2009) 137-162.

[40] E. Garboczi, Computational materials science of cement-based materials, Materials and Structures 26 (4) (1993) 191-195.

[41] G. Constantinides, F. Ulm, K. Van Vliet, On the use of nanoindentation for cementitious materials, Materials and Structures 36 (257) (2003) 191196.

[42] J. Tapli, A method for following the hydration reaction in portland cement paste, Australian Journal of Applied Science 10 (1959) 329-345.

[43] M. Vandamme, The nanogranular origin of concrete: A nanoindentation investigation of microstructure and fundamental properties of calciumsilicate-hydrates, Ph.D. thesis, Massachusetts Institute of Technology (2008).

[44] L. Dormieux, D. Kondo, F.-J. Ulm, Microporomechanics, Wiley, 2006.

[45] V. Levin, Thermal expansion coefficient of heterogeneous materials, Mekh. Tverd. Tela 2 (1967) 83-94.

[46] N. Laws, On the thermostatics of composite materials, Journal of the Mechanics and Physics of Solids 21 (1) (1973) 9-17.

[47] J. D. Eshelby, The determination of the elastic field of an ellipsoidal inclusion, and related problems, Proceedings of the Royal Society of London Series A - Mathematical and Physical Sciences 241 (1226) (1957) 376396.

[48] S. Nemat-Nasser, M. Hori, Micromechanics: Overall properties of heterogeneous materials, Vol. 47, Applied Mathematics and Mechanics, 1993.

[49] A. Stroud, Approximate calculation of multiple integrals, Prentice-Hall, 1971.

[50] T. Mori, K. Tanaka, Average stress in matrix and average elastic energy of materials with misfitting inclusions, Acta Metallurgica 21 (5) (1973) 571-574.

[51] B. Pichler, C. Hellmich, Upscaling quasi-brittle strength of cement paste and mortar: A multi-scale engineering mechanics model, Cement and Concrete Research 41 (5) (2011) 467-476.

[52] G. Constantinides, F.-J. Ulm, The nanogranular nature of C-S-H, Journal of the Mechanics and Physics of Solids 55 (1) (2007) 64-90.

[53] M. Berveiller, B. Sudret, M. Lemaire, Stochastic finite element : a non intrusive approach by regression, Eur. J. Comput. Mech. 15 (2006) 8192.

[54] Sudret, B., Uncertainty propagation and sensitivity analysis in mechanical models - Contributions to structural reliability and stochastic spectral methods, Habilitation à diriger des recherches, Université Blaise Pascal, Clermont-Ferrand, France (2007)

[55] G. Blatman, Adaptive sparse polynomial chaos expansions for uncertainty propagation and sensitivity analysis, Ph.D. thesis, Université Blaise Pascal - Clermont II (2009).

[56] M. Wand, M. Jones, Kernel smoothing, Chapman and Hall, 1995.

[57] B. Sudret, Global sensitivity analysis using polynomial chaos expansions, Reliability Engineering \& System Safety 93 (7) (2008) 964-979.

[58] G. Blatman, B. Sudret, Efficient computation of global sensitivity indices using sparse polynomial chaos expansions, Reliability Engineering System Safety 95 (11) (2010) 1216-1229.

[59] I. Sobol', Sensitivity estimates for nonlinear mathematical models, Math- 
ematical modeling and computational experiment 1 (1993) 407-414.

[60] L. Aldridge, Accuracy and precision of phase analysis in portland cement by Bogue, microscopic and X-ray diffraction methods, Cement and Concrete Research 12 (3) (1982) 381-398.

[61] G. Idorn, A discussion of the paper "Accuracy and precision of phase analyses in Portland cement by Bogue, microscopic and X-ray diffraction methods", by L.P. Aldridge, Cement and Concrete Research 13 (6) (1983) 895.

[62] W. Gutteridge, A discussion of the paper "Accuracy and precision of phase analyses in Portland cement by Bogue, microscopic and X-ray diffraction methods", by L.P. Aldridge, Cement and Concrete Research 13 (6) (1983) 896.

[63] L. Aldridge, A reply to the discussions by G.M. Idorn and W.A. Gutteridge of "Accuracy and precision of phase analysis in Portland cement by Bogue, microscopic and X-ray diffraction methods", Cement and Concrete Research 13 (6) (1983) 897-898.

[64] R. Berliner, M. Popovici, K. Herwig, M. Berliner, H. Jennings, J. Thomas, Quasielastic neutron scattering study of the effect of water-to-cement ratio on the hydration kinetics of tricalcium silicate, Cement and Concrete Research 28 (2) (1998) 231-243.

[65] P. Acker, Micromechanical analysis of creep and shrinkage mechanisms, Creep, Shrinkage and Durability Mechanics of Concrete and Other QuasiBrittle Materials. Edited by F.J. Ulm, Z.P. Bazant and F.-H. Wittman (2001) 15-25.

[66] K. Velez, S. Maximilien, D. Damidot, G. Fantozzi, F. Sorrentino, Determination by nanoindentation of elastic modulus and hardness of pure constituents of portland cement clinker, Cement and Concrete Research 31 (4) (2001) 555-561.

[67] M. Choy, W. Cook, R. Hearmon, J. Jaffe, J. Jerphagon, S. Kurtz, S. Liu, D. Nelson, Landolt-Bornstein: Numerical data and functionnal relationships in science and technology. Group III. Crystal and solid state physics. Vol. 11. Elastic, piezoelectric, pyroelectric, piezooptic, electroptic constants and nonlinear dielectric susceptibilities of crystals, Revised and Extended Edition of Vols III/1 and III/2, Springer-Verlag-Berlin and Heidelberg GmbH \& Co. K, 1979.

[68] A. Bhalla, W. Cook, R. Hearmon, J. Jerphagnon, S. Kurtz, S. Liu, D. Nelson, J.-L. Oudar, Landolt-Bornstein: Numerical data and functionnal relationships in science and technology. Group III. Crystal and solid state physics. Vol. 11. Elastic, piezoelectric, pyroelectric, piezooptic, electroptic constants and nonlinear dielectric susceptibilities of crystals, Supplement to Volume III/11, Springer-Verlag Berlin and Heidelberg GmbH \& Co. K, 1984.

[69] S. Kamali, M. Moranville, E. Garboczi, S. Prené, B. Gérard, Hydrate dissolution influence on the young modulus of cement paste, FRAMCOS $\mathrm{V}$ : Linking scales, from nanostructure to infrastructures (2004) 631-638.

[70] H. M. Jennings, J. J. Thomas, J. S. Gevrenov, G. Constantinides, F. J. Ulm, A multi-technique investigation of the nanoporosity of cement paste, Cement and Concrete Research 37 (3) (2007) 329-336.

[71] M. Berveiller, Éléments finis stochastiques: approches intrusive et non intrusive pour des analyses de fiabilité, Ph.D. thesis, Université Blaise Pascal - Clermont II (2005).

[72] G. Blatman, B. Sudret, An adaptive algorithm to build up sparse polynomial chaos expansions for stochastic finite element analysis, Probabilistic Engineering Mechanics 25 (2) (2010) 183-197. 